ARTICLE

https://doi.org/10.1038/s41467-019-09055-7

\title{
Dietary fatty acids fine-tune Piezo1 mechanical response
}

\author{
Luis O. Romero (1) 1, Andrew E. Massey², Alejandro D. Mata-Daboin', Francisco J. Sierra-Valdez ${ }^{1,3,4}$, \\ Subhash C. Chauhan (1) ${ }^{2}$, Julio F. Cordero-Morales (i) ${ }^{1} \&$ Valeria Vásquez (iD $^{1}$
}

Mechanosensitive ion channels rely on membrane composition to transduce physical stimuli into electrical signals. The Piezo1 channel mediates mechanoelectrical transduction and regulates crucial physiological processes, including vascular architecture and remodeling, cell migration, and erythrocyte volume. The identity of the membrane components that modulate Piezo1 function remain largely unknown. Using lipid profiling analyses, we here identify dietary fatty acids that tune Piezol mechanical response. We find that margaric acid, a saturated fatty acid present in dairy products and fish, inhibits Piezo1 activation and polyunsaturated fatty acids (PUFAs), present in fish oils, modulate channel inactivation. Force measurements reveal that margaric acid increases membrane bending stiffness, whereas PUFAs decrease it. We use fatty acid supplementation to abrogate the phenotype of gain-offunction Piezo1 mutations causing human dehydrated hereditary stomatocytosis. Beyond Piezo1, our findings demonstrate that cell-intrinsic lipid profile and changes in the fatty acid metabolism can dictate the cell's response to mechanical cues.

\footnotetext{
${ }^{1}$ Department of Physiology, College of Medicine, University of Tennessee Health Science Center, 71S. Manassas St., Memphis, TN 38163, USA. ${ }^{2}$ Department of Pharmaceutical Sciences and Institute of Biomarker and Molecular Therapeutics (IBMT), College of Pharmacy, University of Tennessee Health Science Center, 881 Madison Ave., Memphis, TN 38163, USA. ${ }^{3}$ Present address: Centro de Investigación Biomédica, Hospital Zambrano Hellion, TecSalud, Ave. Batallon de San Patricio 112, 66278 San Pedro Garza García, Nuevo León, Mexico. ${ }^{4}$ Present address: Tecnólogico de Monterrey, Escuela de Ingeniería y Ciencias, Ave. Eugenio Garza Sada 2501 Sur, 64849 Monterrey, Nuevo León, Mexico. Correspondence and requests for materials should be addressed to V.V. (email: vvasquez@uthsc.edu)
} 
M echanosensitive ion channels are membrane proteins that translate mechanical stimuli into electrical signals that lead to physiological responses. The Piezol mechanosensitive cation channel has been shown to mediate crucial physiological processes that include, but are not limited to, blood pressure regulation ${ }^{1-3}$, vascular architecture and remodeling ${ }^{4,5}$, erythrocyte volume regulation ${ }^{6}$, chondrocyte mechanotransduction ${ }^{7,8}$, lineage choice in neural stem cells ${ }^{9}$, and association of neurons with astrocytes ${ }^{10}$. Piezol is important in mammals, as global knockouts are embryonic lethal in mice ${ }^{4,5}$, and cell-specific knockouts result in animals with severe defects ${ }^{11,12}$. Cells continuously facing mechanical cues such as shear and osmotic stress, stretch, and changes in substrate stiffness rely on the fast response (milliseconds) of Piezol to regulate cellular processes that occur on larger timescales (seconds to days $)^{11,13}$. Consequently, Piezo1 gain- and loss-of-function mutants have been associated with several hereditary human pathophysiologies ${ }^{14,15}$. For example, some mutations in Piezol are linked to inherited blood disorders (e.g., dehydrated hereditary stomatocytosis or xerocytosis ${ }^{16-19}$ ), in which the mutant channel displays slow inactivation ${ }^{20}$, leading to an increase in cation permeability and subsequent dehydrated erythrocytes ${ }^{12}$. To better understand Piezol role in physiological and pathophysiological conditions, it is essential to determine the proteins and molecules that regulate its function.

While it is widely agreed that Piezol is activated by membrane tension ${ }^{21-24}$, little is known about the role of membrane lipids 25,26 ; particularly, whether fatty acids modulate Piezo1 function. Dietary fatty acids are among the membrane lipid components that dynamically regulate ion channel function ${ }^{27,28}$. The most common fatty acids in membrane lipids have 14-22 carbon atoms and carry one unsaturation (e.g., oleic acid), and in some cases as many as six double bonds ${ }^{29}$. These chemical features confer cells the capacity to control membrane thickness and fluidity in different physiological contexts. Polyunsaturated fatty acids (PUFAs), in particular, regulate plasma membrane remodeling by endowing the membrane with distinct elastic proper$\operatorname{ties}^{27}$. For instance, the function of TRP and TRP-like photomechanical receptors in Drosophila melanogaster is modulated by fatty acid-containing phospholipids, as increasing the content of saturated fatty acids in the diet leads to a decline in the electrical light response associated with these channels ${ }^{30}$. Likewise, plasma membranes containing the $\omega-3$ PUFA eicosapentaenoic acid (EPA) and its eicosanoid derivative 17,18-EEQ enhance TRPV4 chemical and osmotic activities in human microvascular endothelial cells (HMVEC) ${ }^{31}$. Given that Piezol is activated by changes in membrane tension, we sought to determine whether changing the mechanical properties of the plasma membrane with different dietary fatty acids would have an effect on Piezol mechanical gating.

Here, we use lipidomics and electrophysiology to demonstrate that Piezo1-mediated mechanocurrents are modulated by dietary fatty acids in several cell lines including: the mouse neuro-2a (N2A) cells, HMVEC, and human embryonic kidney (HEK-293) cells. We find that when part of the plasma membrane, the saturated fatty acid margaric acid (MA; C17) inhibits Piezo1 currents, whereas C20 (arachidonic acid [AA] and EPA) and C22 (docosahexaenoic acid, DHA) PUFAs regulates inactivation. Differential scanning calorimetry (DSC) and atomic force microscopy (AFM) experiments show that membranes enriched in MA are more rigid and display higher bending stiffness than those containing PUFAs. N2A cells and HMVEC display distinct fatty acid profiles as well as Piezol different inactivation properties. We demonstrate here that culturing N2A cells with a fatty acid enriched in HMVEC changes Piezol to feature currents similar to the ones found in HMVEC. Finally, we determine that fatty acids supplementation reverses the slow inactivation phenotype of Piezol gain-of-function mutations associated with human dehydrated hereditary stomatocytosis. Our findings demonstrate that saturated and PUFAs contained in the plasma membrane modulate the mechanical response of Piezol.

\section{Results}

Margaric acid inhibits N2A endogenous Piezol currents. Piezol channels were first identified by mechanically stimulating N2A cells using a piezo-electrically driven glass probe ${ }^{32}$. To determine how common dietary fatty acids modulate Piezol's mechanical response in N2A cells, we characterized their membrane fatty acid profile using liquid chromatography-mass spectrometry (LC-MS). We found basal levels of the odd-chain saturated fatty acids MA (C17:0) and pentadecanoic acid (PDA; C15:0) (Supplementary Figure 1a, red). We hypothesized that increasing the amount of such fatty acids might alter membrane fluidity and in consequence modify Piezol activity. To this end, we performed whole-cell patch-clamp recordings (at $-60 \mathrm{mV}$ ) after supplementing the N2A cell media with saturated fatty acids ranging between 1 and $300 \mu \mathrm{M}$ for $18 \mathrm{~h}$ (overnight). These concentrations are within the range of circulating fatty acids present in blood plasma of healthy adults ${ }^{33}$. Piezol consistently displays robust and fast inactivating currents $(\tau \approx 33.8 \mathrm{~ms}$, at $-60 \mathrm{mV})$ under mechanical displacement (Fig. 1a, control, and elsewhere $^{32}$ ). Remarkably, we found that MA inhibits Piezol currents in a concentration-dependent manner (Fig. 1a, b), while retaining the time constants of inactivation of the non-treated cells (Supplementary Figure $1 \mathrm{~b}$ ). The magnitude of Piezol currents decreases with an $\mathrm{IC}_{50}=28.3 \pm 3.4 \mu \mathrm{M}$ (mean \pm SEM; Fig. 1b). With high concentrations of MA $(\geq 50 \mu \mathrm{M})$, the likelihood of activating Piezol decreases (Fig. 1a and Table 1 for $100 \mu \mathrm{M}, 6$ out of 13 cells did not display activity), even when poking the N2A cells to the limit of patch-clamp rupture.

We also used an alternative supplementation protocol consisting of adding lower doses of MA (insufficient to inhibit the channel) for several days. Supplementing N2A cells with $10 \mu \mathrm{M}$ MA each day, for 5 days, significantly decreased Piezo1 currents (Fig. 1c). We used LC-MS to quantify the incorporation of MA in N2A membranes after fatty acid supplementation. The MA content increased 30- and 8-fold in cells treated with $100 \mu \mathrm{M}$ MA overnight or $10 \mu \mathrm{M}$ for 5 days, respectively, with respect to the control cells (Fig. 1d). On the other hand, supplementing N2A cells with PDA did not alter Piezol channel function (Supplementary Figure $1 \mathrm{c}-\mathrm{f}$ ) even though it was enriched 37 -fold (Supplementary Figure 1g). As MA and PDA have similar structures but differ in their acyl-chain length (Fig. 1a and Supplementary Figure 1c), our results support the idea that saturated fatty acids with $>15$ carbons impair the mechanical response of Piezol.

Next, we asked whether the inhibitory effect of MA is due to a decrease in Piezol channel function rather than a lack of channel expression or trafficking. Yoda1, known to sensitize Piezol to mechanical stimuli ${ }^{34}$, recovered Piezol currents in MA $(100 \mu \mathrm{M})$ treated cells that could not be activated by displacement alone (Fig. 1a, e). This result supports the idea that MA shifts Piezo1 activation curve with no effect in channel expression or trafficking. Indeed, the displacement threshold for eliciting Piezo1 currents in MA (100 and $300 \mu \mathrm{M})$-supplemented N2A cells was twofold when compared to that of the control (Fig. 1f). Although indentation generates variable stimulus-response relationships ${ }^{11}$, we consistently required high displacement steps to elicit Piezol currents in the presence of MA. As expected, addition of Yodal prior mechanical stimulation lowered the displacement threshold, similar to the one obtained for the 
a [Margaric acid] $(\mu \mathrm{M})$ Control MA, 17:0

N2A cells

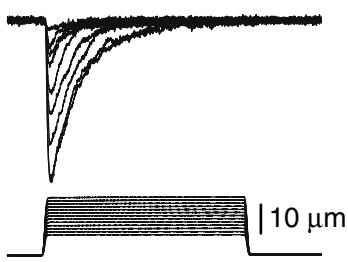

50
1

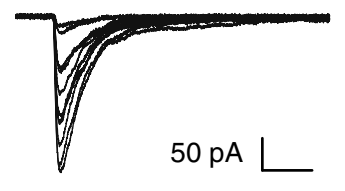

$50 \mathrm{~ms}$
100

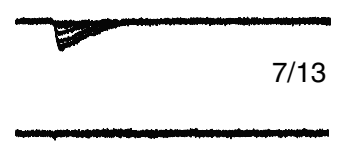

$6 / 13$
10

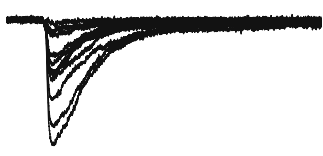

300
$\mathrm{MA}(100 \mu \mathrm{M})+$

Yoda1 $(15 \mu \mathrm{M})$
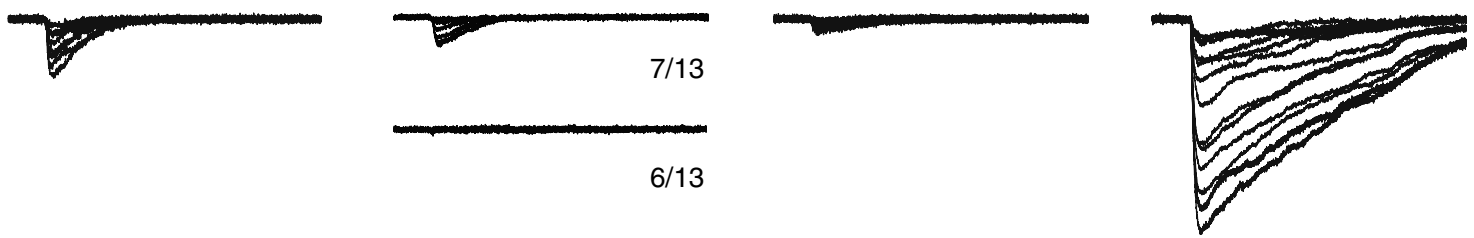

b

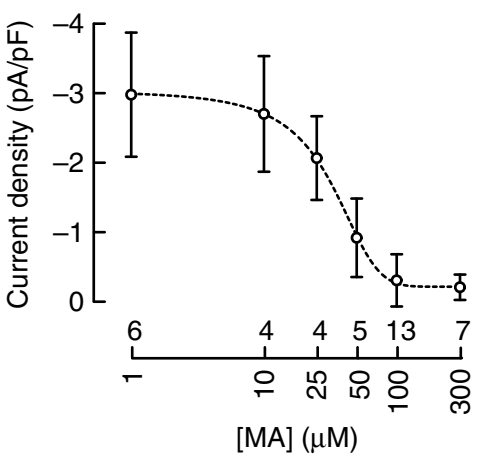

C

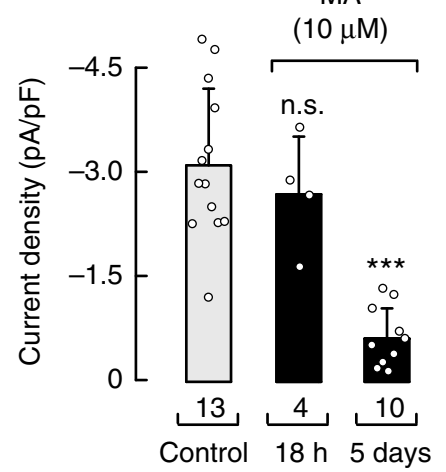

d

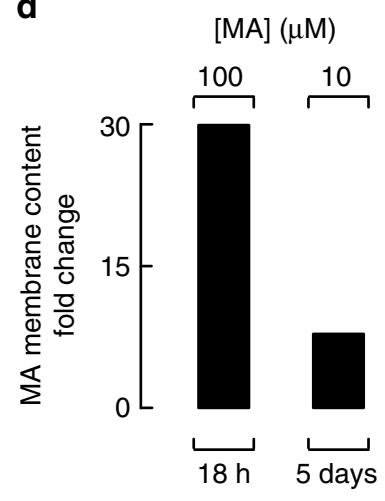

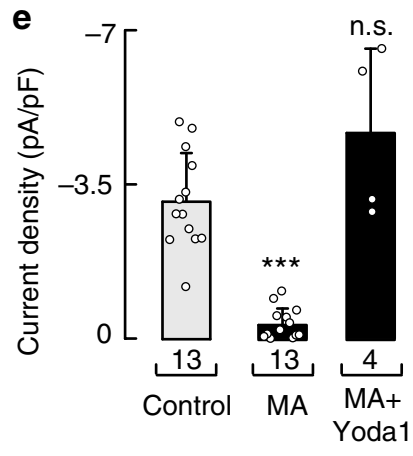
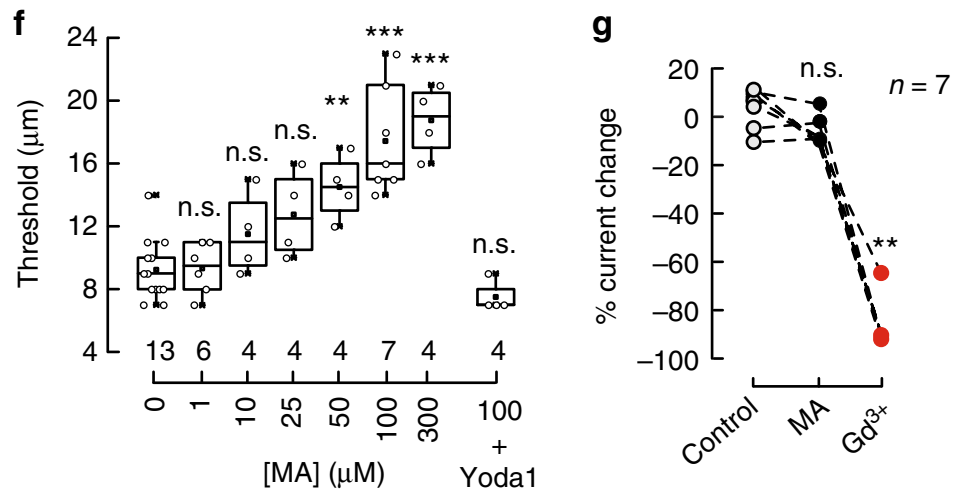

Fig. 1 Margaric acid inhibits Piezo1 currents in N2A cells. a Representative whole-cell patch-clamp recordings (at $-60 \mathrm{mV}$ ) of control and margaric acid (MA) $(1,10,25,50,100$, and $300 \mu M)$-treated N2A cells elicited by mechanical stimulation. Bottom right panel displays representative Piezo1 macroscopic currents of MA-treated N2A cells incubated with Yoda1 prior to mechanical stimulation. The structure of MA is displayed on top. $\mathbf{b}$ Piezo1 current densities elicited by maximum displacement of MA-treated N2A cells. A Boltzmann function, Eq. (2), was fitted to the data $\left(I C_{50}=28.3 \pm 3.4\right.$ SEM). Circles are mean \pm SD. $n$ is denoted above the $x$-axis. c Piezo1 current densities elicited by maximum displacement of control and MA (10 $\mu$ M; $18 \mathrm{~h}$ and 5 days)-treated N2A cells. $n$ is denoted above the $x$-axis. Kruskal-Wallis and Dunn's multiple comparisons test. $\mathbf{d}$ MA membrane content fold change in N2A cells treated with MA $100 \mu \mathrm{M}$ for $18 \mathrm{~h}$ or $10 \mu \mathrm{M}$ each day for 5 days, as determined by LC-MS. e Piezo1 current densities elicited by maximum displacement of control, MA $(100 \mu \mathrm{M})$-treated N2A cells, and MA $(100 \mu \mathrm{M})$-treated N2A cells incubated with $15 \mu \mathrm{M}$ Yoda1 prior to mechanical stimulation. Bars are mean \pm SD. $n$ is denoted above the $x$-axis. Kruskal-Wallis and Dunn's multiple comparisons test. $\mathbf{f}$ Boxplots show the mean, median, and the 75th to 25th percentiles of the displacement thresholds required to elicit Piezo1 currents of control and MA-treated N2A cells. $\mathrm{n}$ is denoted above the $x$-axis. One-way ANOVA and Bonferroni test. $\mathbf{g}$ Current changes elicited by maximum displacement of N2A cells perfused for $120 \mathrm{~s}$ with bath solution (control), MA (100 $\mu \mathrm{M})$, and $\mathrm{Gd} \mathrm{d}^{3+}$ $(30 \mu \mathrm{M})$ consecutively. $\mathrm{Gd}^{3}+$ was used as control for the perfusion. Data samples are paired. $n$ is denoted above the plot. Friedman test. Asterisks indicate values significantly different from control $\left({ }^{* *} p<0.001\right.$ and $\left.{ }^{* *} p<0.01\right)$ and n.s. indicates not significantly different from the control 
control (Fig. 1f). Interestingly, perfusion of $100 \mu \mathrm{M}$ MA in solution to $\mathrm{N} 2 \mathrm{~A}$ cells while poking under the whole-cell configuration did not inhibit Piezol currents (Fig. $1 \mathrm{~g}$ and Supplementary Figure 1h), suggesting that the effect of MA could be due to membrane remodeling rather than a direct effect.

\section{Table 1 Proportion of N2A cells that display Piezo1 currents after MA supplementation}

\begin{tabular}{ll} 
[Margaric acid] $(\boldsymbol{\mu M})$ & \% of N2A cells with Piezo1 currents \\
\hline 0 & 100 \\
1 & 100 \\
10 & 100 \\
25 & 100 \\
50 & 80 \\
100 & 54 \\
300 & 57 \\
$100+$ Yoda1 & 100
\end{tabular}

Taken together, our results demonstrate that MA treatment inhibits Piezol currents by increasing the mechanical threshold required for activation.

PUFAs modulate Piezo1 inactivation. Long $\omega-6$ and $\omega-3$ PUFAs $(\geq \mathrm{C} 20)$ - enriched in meat and fish, respectively-are known to increase membrane structural disorder, decrease stiffness, and alter ion channel function $27,31,35-38$. To determine the role of long PUFAs in Piezol mechanical gating, we supplemented the N2A cell media with $\omega-6$ AA (C20:4), $\omega-3$ EPA (C20:5), and $\omega-3$ DHA (C22:6). We found that C20 PUFAs decreased Piezol time constants of inactivation to $\approx 20 \mathrm{~ms}(100 \mu \mathrm{M}$ of AA and EPA, Fig. 2a, b) compared to control cells $(\approx 34 \mathrm{~ms})$. Moreover, we observed a further decrease in the time constants of inactivation ( $\approx 13 \mathrm{~ms}$ ) when supplementing N2A cells with higher EPA concentrations (200 and $300 \mu \mathrm{M}$, Supplementary Figure 2a, b). Interestingly, supplementing $\mathrm{N} 2 \mathrm{~A}$ cells with low concentrations $(10 \mu \mathrm{M})$ of EPA, each day for 5 days, increases the EPA content in the plasma membrane and significantly speed up Piezol a

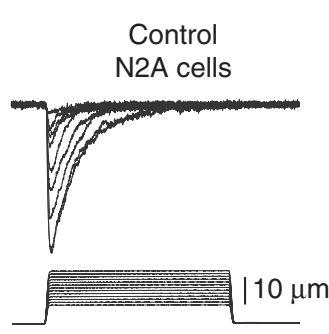

b

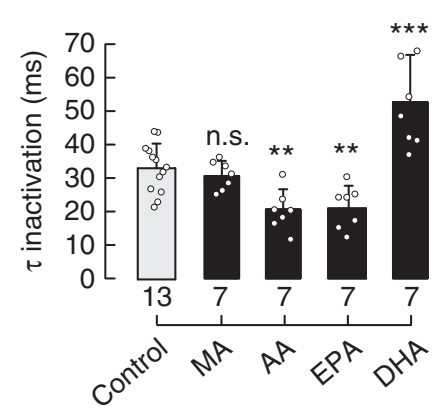

e

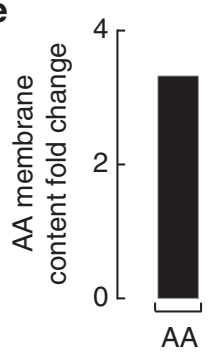

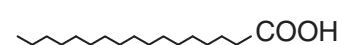

Margaric acid (MA) 17:0

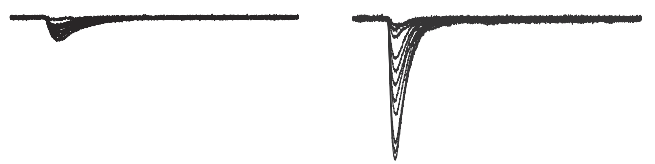

$50 \mathrm{pA}$

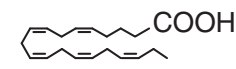

Eicosapentaenoic acid (EPA) 20:5

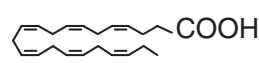

Docosahexaenoic acid (DHA) 22:6
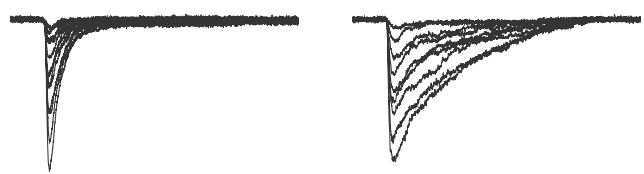

$$
50 \mathrm{~ms}
$$

C

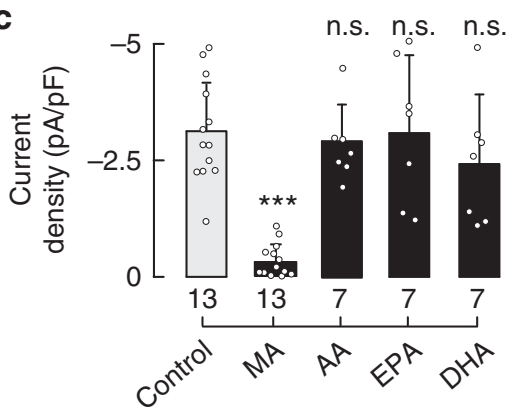

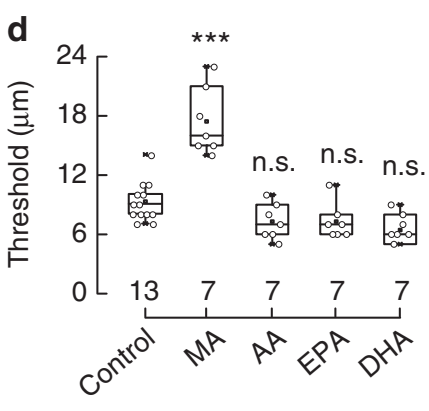

f

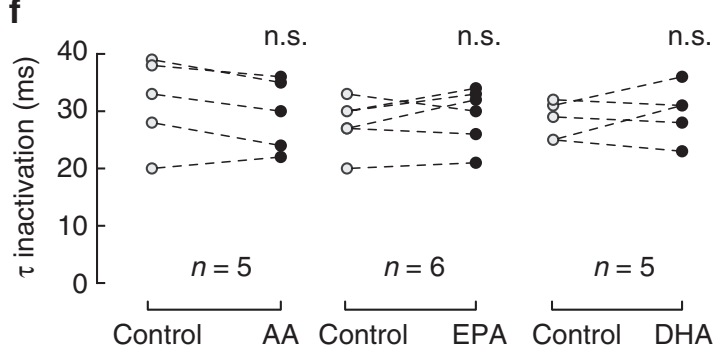

Fig. 2 Dietary fatty acids alter Piezo1 channel gating. a Representative whole-cell patch-clamp recordings (at $-60 \mathrm{mV}$ ) of control, MA, AA, EPA, and DHA $(100 \mu \mathrm{M})$-treated N2A cells elicited by mechanical stimulation. The structures of MA, AA, EPA, and DHA are displayed above. $\mathbf{b}$ Piezo1 time constants of inactivation elicited by maximum displacement of control, MA, AA, EPA, and DHA (100 $\mu M)$-treated N2A cells. Bars are mean \pm SD. One-way ANOVA and Bonferroni test. c Current densities elicited by maximum displacement of control, MA, AA, EPA, and DHA (100 $\mu$ M)-treated N2A cells. Bars are mean \pm SD. Kruskal-Wallis and Dunn's multiple comparisons test. $\mathbf{d}$ Boxplots show the mean, median, and the 75th to 25th percentiles of the displacement threshold required to elicit Piezo1 currents of control, MA, AA, EPA, and DHA (100 $\mu M)$-treated N2A cells. One-way ANOVA and Bonferroni test. e AA, EPA, and DHA membrane content fold change in N2A cells treated with AA, EPA, and DHA $100 \mu \mathrm{M}$ for $18 \mathrm{~h}$, as determined by liquid chromatography-mass spectrometry (LC-MS). f Piezo1 time constants of inactivation elicited by maximum displacement of N2A cells perfused for $120 \mathrm{~s}$ with bath solution (control) and a PUFA (AA, EPA, and DHA; $100 \mu \mathrm{M}$ ). Data samples are paired. Paired $t$-test. Asterisks indicate values significantly different from control $\left({ }^{* * *} p<0.001\right.$ and $\left.{ }^{* *} p<0.01\right)$ and n.s. indicates not significantly different from the control. $n$ is denoted above the $x$-axes 
a

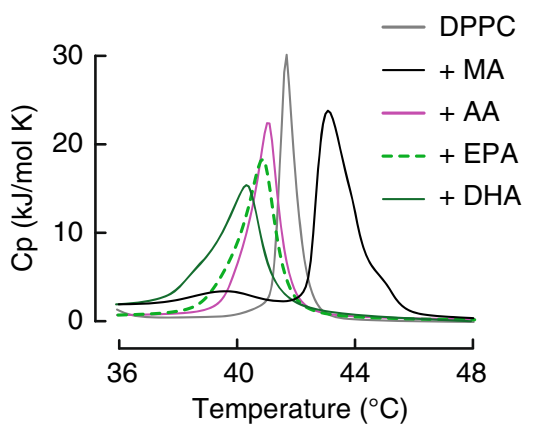

b

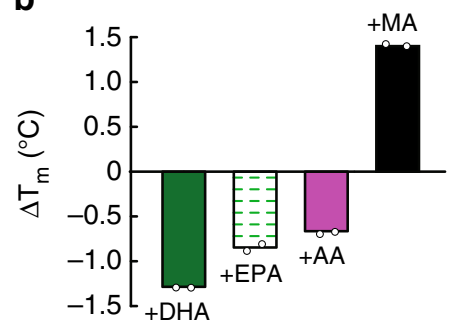

C
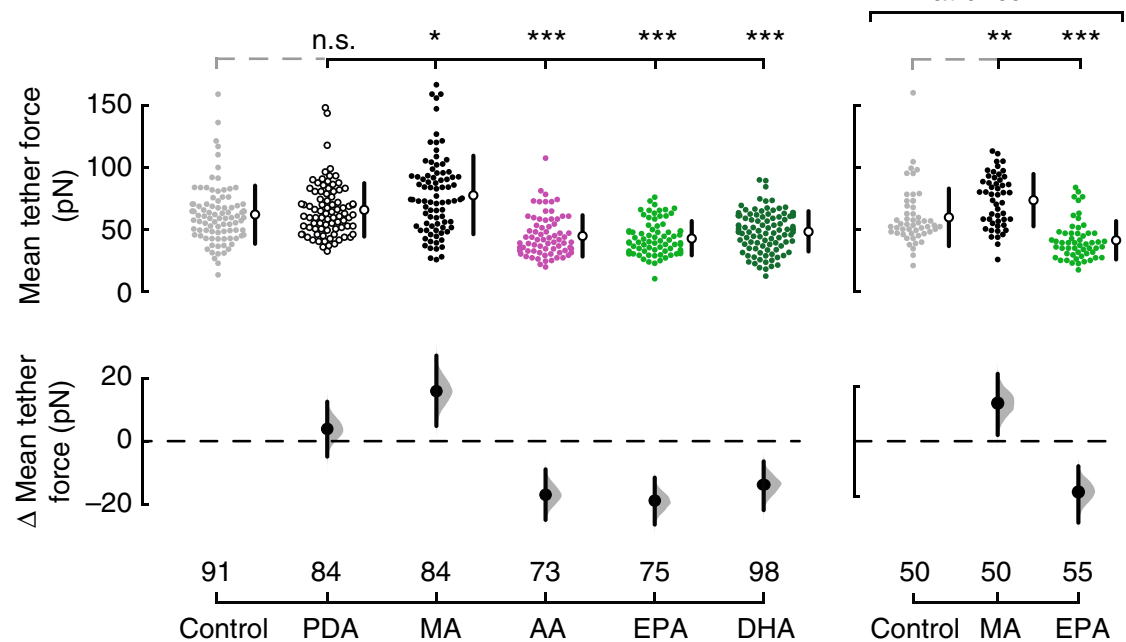

Fig. 3 Dietary fatty acids alter membrane fluidity in N2A cells. a Representative thermotropic characterization of the DPPC/fatty acid systems using DSC: control $\left(T_{m}=41.68^{\circ} \mathrm{C}\right), \mathrm{MA}\left(43.08^{\circ} \mathrm{C}\right), \mathrm{AA}\left(41.04^{\circ} \mathrm{C}\right), \mathrm{EPA}\left(40.85^{\circ} \mathrm{C}\right)$, and DHA $\left(40.40^{\circ} \mathrm{C}\right)$. $\mathbf{b}$ Effects of DPPC/fatty acids on melting temperatures $\left(\Delta T_{\mathrm{m}}\right)$ with respect to DPPC membranes. Experiments were performed from two independent preparations. $\mathrm{c}$ Estimation plot with all tether force data points presented as a swarmplot for control, pentadecanoic acid (PDA), MA, AA, EPA, and DHA (100 $\mu M)$-treated N2A cells. $\mathbf{d}$ Estimation plot with all tether force data points presented as a swarmplot for latrunculin $A(1 \mu \mathrm{M} ; 1 \mathrm{~h})$-treated N2A control cells and supplemented with MA (100 $\mu \mathrm{M})$ or EPA $(100 \mu \mathrm{M})$. Bootstraps with $99.9 \%$ confidence interval and a comparison with the mean of the control group are displayed to the right and bottom of the raw data, respectively. Kruskal-Wallis and Dunn's multiple comparisons test. Asterisks indicate values significantly different from control $(* * * p<0.001$, ${ }^{*} p<0.01$, and ${ }^{*} p<0.05$ ) and n.s. indicates not significantly different from the control. $n$ is denoted above the $x$-axes

inactivation $(\approx 13 \mathrm{~ms}$; Supplementary Figure $2 \mathrm{a}-\mathrm{c})$. On the other hand, C22 DHA elicited a robust increase in the time constants of inactivation up to $\approx 53 \mathrm{~ms}(100 \mu \mathrm{M}$, Fig. $2 \mathrm{a}, \mathrm{b})$. We did not find differences in current magnitudes or displacement thresholds with these long PUFAs when compared to those of the control (Fig. 2c, d). It is remarkable that the $\omega-3$ fatty acids EPA and DHA elicited distinct effects on inactivation even though their structures are similar (Fig. 2a, top). We also determined that PUFAs (AA, EPA, and DHA) are efficiently incorporated in N2A membranes (Fig. 2e). Our results suggest that saturated fatty acids (like MA) control the extent of Piezol activation, and once Piezo1 is open long PUFAs determine the time course of its inactivation. Similar to MA, when directly perfused while mechanically stimulating N2A cells, long PUFAs did not have an effect on Piezo1 inactivation or current magnitudes (Fig. $2 \mathrm{f}$ and Supplementary Figure 2d). Hence, we hypothesized that fatty acids modulate Piezol's activity by changing the physical properties of the plasma membrane (e.g., structural disorder and stiffness).

Fatty acids alter Piezo1 via the plasma membrane. Using DSC, we measured changes in heat-capacity profiles $\left(C_{\mathrm{p}}\right)$ as a readout for membrane structural disorder of synthetic membranes (1,2dipalmitoyl-sn-glycero-3-phosphocholine, DPPC) with and without fatty acids. We found that MA evokes a robust shift in the melting temperature $\left(T_{\mathrm{m}}\right)$ toward higher values indicative of an increase in membrane structural order $\left(\Delta T_{\mathrm{m}}=1.39\right.$; Fig. 3a, b, black). On the other hand, long PUFAs increase membrane structural disorder and decrease cooperativity among lipids, as observed by the leftward shift and broadening of the main melting peaks corresponding to DHA, EPA, and AA (Fig. 3a, b, dark and light greens, and magenta, respectively). The DSC results in synthetic lipids suggest that fatty acids endow membranes with distinct structural properties that might determine the magnitude and extent of Piezol's mechanical response.

Measurements of tether force using AFM allow determination of the effective plasma membrane fluidity and bending stiffness in live cells ${ }^{31,37}$. We have previously determined that plasma membranes enriched in EPA feature lower bending stiffness than control cells ${ }^{31,37}$. To directly determine the extent by which PDA, MA, AA, EPA, and DHA alter the mechanical properties of N2A plasma membranes, we measured the mean tether forces of control and fatty acid-treated cells. The mean tether force for control N2A cells $(61.9 \pm 23.4 \mathrm{pN}$, mean \pm SD; Fig. $3 \mathrm{c})$ is similar to the ones reported for other cultured cells and neurons $31,37,39$. We found that MA treatment significantly increased the mean tether force $(77.8 \pm 31.5 \mathrm{pN}$, mean $\pm \mathrm{SD})$ when compared to untreated cells (Fig. 3c and Supplementary Figure 3a), indicating 
that plasma membranes enriched in MA display high bending stiffness. Importantly, treatment with PDA did not alter the elastic properties of $\mathrm{N} 2 \mathrm{~A}$ plasma membranes $(65.8 \pm 21.5$; mean \pm SD; Fig. $3 c$ ); this property correlates with its inability to modify Piezo1 mechanical response (Supplementary Figure 1c-f). On the other hand, C20 and C22 PUFAs had the opposite effect (low bending stiffness) on plasma membranes as they significantly decreased their mean tether force $(44.9 \pm 16.6$ and $43 \pm 13.8,48.5 \pm 16.2$, mean $\pm \mathrm{SD}, \mathrm{AA}, \mathrm{EPA}$ and DHA, respectively; Fig. 3c).

To evaluate the contribution of the cytoskeleton to the mean tether force of membranes enriched with specific fatty acids, we disrupted actin polymerization using latrunculin A. Cells treated with latrunculin A display round shape with less filopodia likeprotrusions (Supplementary Figure $3 \mathrm{~b}$ ). We found that this treatment did not have an effect on the mean tether forces of N2A plasma membranes $(61.9 \pm 23.4$ control vs. $59.7 \pm 23.1$ latrunculin A, mean \pm SD; Fig. 3c, d, light gray circles). Moreover, disrupting the actin filaments did not change the effect that fatty acids enrichment had on the plasma membrane mechanics, as MA increased the mean tether force $(73.6 \pm 21$, mean $\pm S D)$ and EPA decreased it $(41.3 \pm 15.4$, mean \pm SD; Fig. $3 \mathrm{~d})$. As expected, in latrunculin A-treated cells, MA inhibits Piezol currents and increases the displacement threshold, and EPA enhances its inactivation (Supplementary Figure 3c-f). Interestingly, disrupting actin polymerization decreases the magnitude of Piezo1 current densities $(-3.11 \pm 1.1$ control vs. $-1.49 \pm 0.5$ latrunculin A-treated N2A cells, mean \pm SD; Fig. 1c and Supplementary Figure $3 \mathrm{~d}$, gray bars). This result suggests that less channels are being recruited with the mechanical probe in cells treated with latrunculin A, likely due to the disrupted cytoskeleton and an inefficient transmission of the mechanical stimuli. Similar results were reported by the Goodman laboratory when measuring mechanoreceptor currents and mean tether forces from neurons lacking cytoskeletal proteins ${ }^{40,41}$. Altogether, our results support that MA decreases Piezol activation by increasing membrane structural order and bending stiffness, whereas PUFAs modify Piezo1 inactivation by decreasing structural order and bending stiffness.

Piezo1 mediates indentation-activated currents in HMVEC. Piezo1 is also expressed in endothelial cells, where it has been shown to gate in response to shear stress ${ }^{4,5}$, enable vascular development ${ }^{4,5}$, mediate flow-induced ATP release $^{1}$, and sense physical activity ${ }^{2}$. Whether Piezo1 mediates indentation-activated currents in HMVEC has not been previously established. HMVEC have a fatty acid distribution profile different from that of N2A cells, highly enriched in saturated fatty acids and $\omega-6$ PUFAs (Fig. 4a, gray and magenta), as determined by LC-MS. In view of the results described above, we anticipated that Piezol in HMVEC would feature channel properties distinct from those measured in N2A cells. To test this idea, we characterized the mechanically activated currents in HMVEC by indenting the cells with 1- $\mu \mathrm{m}$-step pulses. We found that HMVEC indentationactivated currents (Fig. 4b) displayed most of the properties described for Piezo1 in other cell types: voltage-dependent inactivation $^{42}$, non-selective cation currents ${ }^{32}$ (reversal potential at $+7.5 \mathrm{mV}$, Fig. $4 \mathrm{c}$ ), complete blockage by $\mathrm{GdCl}_{3}{ }^{32}$, and partial inhibition by L-GsMTX- ${ }^{20}$ (Supplementary Figure 4a). Moreover, specific blockers of other cation channels (e.g., TRPC3, TRPC6, TRPV4, and TRPA1) did not decrease the magnitude of the indentation-mediated currents (Supplementary Figure $4 \mathrm{~b}$ ). It is noteworthy that the large steady-state currents $(\approx 50 \%$ of the peak, red arrow) observed in most of our whole-cell recordings seem to be a feature for HMVEC mechano-activated currents
(Fig. 4b). Furthermore, we determined that Piezol is functional in HMVEC when measuring a significant increase in the fluorescence signal after addition of Yodal to cells loaded with a $\mathrm{Ca}^{2+}$-sensitive dye (Fluo-4 AM; Fig. 4d and Supplementary Figure 5a). Finally, knocking down the expression of Piezol with silencing RNA (siRNA) abolished, almost completely, the indentation-mediated currents of HMVEC (Fig. 4e and Supplementary Fig. 5b-f). Overall, our results demonstrate that Piezo1 mediates indentation-activated currents in HMVEC and displays distinct mechano currents likely due to differences in the fatty acids profile.

Fatty acids alter HMVEC Piezol channel gating. We asked whether Piezo1 gating modulation by fatty acids in N2A cells was only a feature of this mouse cell line or could occur in other cell types. To this end, we measured the effects of MA and long PUFAs on Piezol activity under an inherently different fatty acid regime like the one found in HMVEC (Fig. 4a). As observed in N2A cells, MA supplementation in HMVEC inhibited Piezo1 currents (Fig. 5a, b) by increasing the displacement threshold (Fig. 5c). Moreover, MA treatment did not alter the ratio of peak to steady-state currents (i.e., inactivation) of Piezol (Fig. 5d). In contrast to MA, C20 and C22 PUFAs have differential effects on Piezo1 inactivation in HMVEC. EPA $(100 \mu \mathrm{M} ; 18 \mathrm{~h})$ slightly diminishes (albeit not significant) the ratio of peak to steady-state currents (Fig. 5a, d); this result was unexpected since EPA strongly enhances the inactivation in N2A cells. One explanation for this result might be that HMVEC membranes require more EPA than N2A cells to modulate Piezo gating. We tested this idea by supplementing HMVEC with low EPA concentrations for several days ( $50 \mu \mathrm{M} ; 3$ days). Indeed, using this supplementation protocol, we found an increase in EPA content (18 h vs. 3 days, Fig. 5e) in HMVEC as well as a significant increase in inactivation (Fig. 5f). Notably, PUFAs did not change current densities or the displacement thresholds (Fig. 5b, c and Supplementary Figure 6a, b). As observed in N2A cells, DHA decrease inactivation in HMVEC (Fig. 5a, d), as the currents at the end of the indentation pulse were $\approx 85 \%$ of the activation peak, while maintaining current densities and displacement thresholds similar to those of the control cells (Fig. 5b, c). These results demonstrate that Piezol modulation by fatty acids is not unique to N2A cells and is independent of the channel ortholog (mouse vs. human). LC-MS analysis demonstrates that in the culture conditions used for electrophysiological recordings, HMVEC are able to incorporate MA, AA, EPA, and DHA in the membranes, albeit to different extents (Fig. 5e, g). Altogether our results, from N2A and HMVEC, show that supplementation of saturated and unsaturated fatty acids could be useful for tuning cells' responses to mechanical stimuli.

Global fatty acid distribution modulates Piezol gating. When analyzing the fatty acid content of N2A cells and HMVEC, we noticed that linoleic acid (C18:2) was higher in HMVEC (Fig. 6a). Linoleic acid - an essential $\omega-6$ fatty acid - is a metabolic precursor for AA; due to its limited conversion by the delta- 6 desaturase enzyme ${ }^{43}$, linoleic acid can accumulate when its consumption in the diet is increased. Indeed, we observed accumulation of linoleic acid in supplemented N2A cells, while keeping the content of downstream AA lower than the precursor (Supplementary Figure 7a, right panel). As Piezo1 inactivation in N2A cells is more prominent than in HMVEC, we wondered whether increasing the content of linoleic acid and its downstream metabolic products would modify Piezol's mechanical response to resemble that of HMVEC. Remarkably, N2A cells supplemented with linoleic acid displayed a global fatty acid 
a
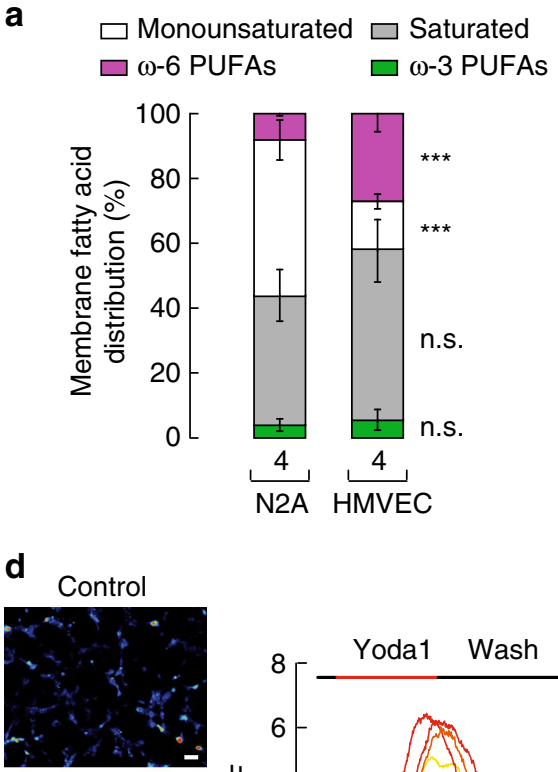

Yoda1

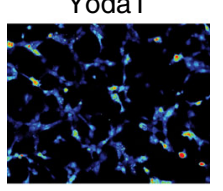

Low $=$ High

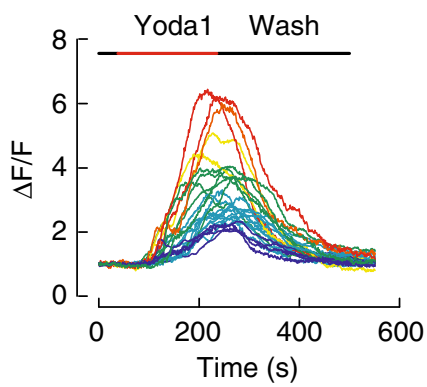

b

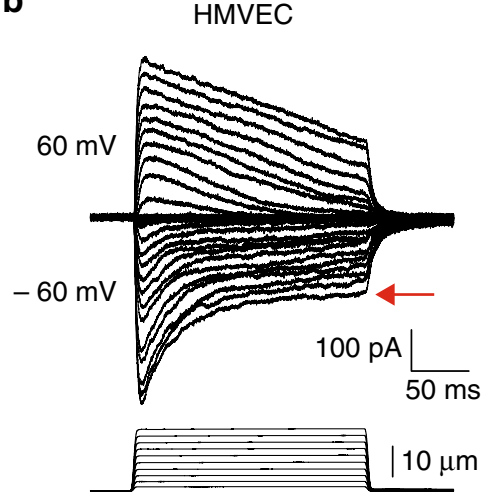

c

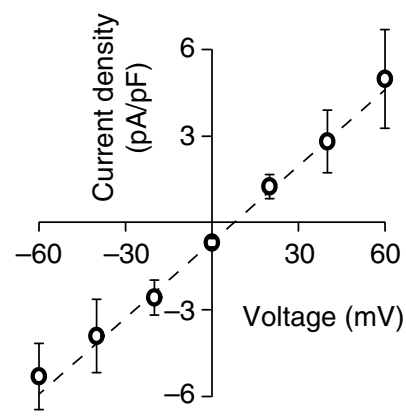

$\mathbf{e}$
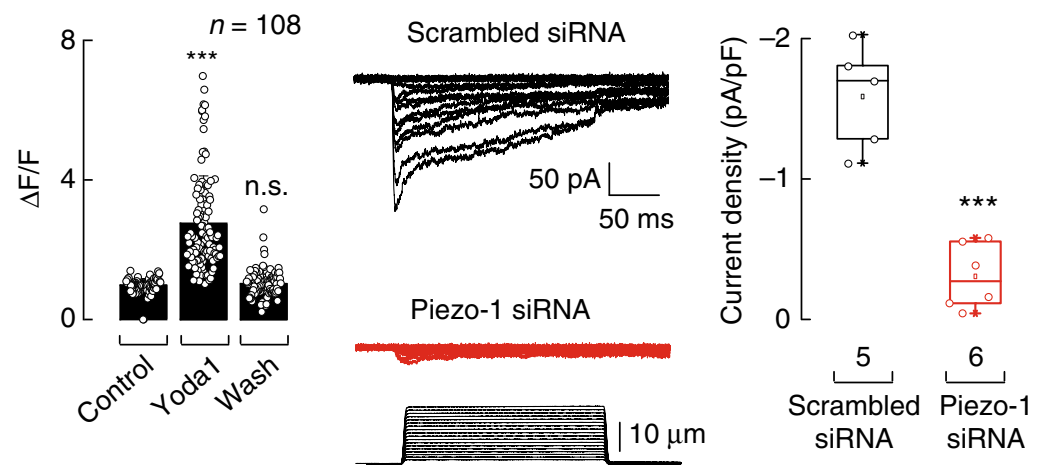

Fig. 4 Piezo1 channel mediates mechanically evoked currents in HMVEC. a Stacked bar chart illustrating the membrane fatty acid distribution in N2A cells and HMVEC, as determined by LC-MS. Bars are mean \pm SD. $n$ is denoted above the $x$-axis. Mann-Whitney test. b Representative whole-cell patch-clamp recordings (at $\pm 60 \mathrm{mV}$ ) of HMVEC (top) elicited by mechanical stimulation (bottom). Red arrow highlights the steady-state currents. c Current-voltage relationship of HMVEC mechano-dependent currents as determined by whole-cell patch-clamp experiments. Circles are mean \pm SD. $n=5$. $\mathbf{d}$ Left: representative micrographs of HMVEC challenged with control buffer and $2 \mu \mathrm{M}$ Yoda1 and analyzed for their responses using Ca2+ imaging (Fluo-4 AM); color bar indicates relative change in fluorescence intensity. White bar represents $50 \mu \mathrm{m}$. Middle: representative traces corresponding to intensity changes $(\Delta F / F)$ of individual cells shown in left panel. Right: mean fluorescence intensity values $(\Delta F / F)$ of HMVEC perfused with control solution $(t=30 \mathrm{~s})$, Yoda1 $(t=250 \mathrm{~s})$, and washed with control solution $(t=500 \mathrm{~s})$. Bars are mean \pm SD. $n$ is denoted above the plot. Friedman test. e Left: representative HMVEC mechano-dependent current densities transfected with scrambled or Piezo1 siRNA elicited by mechanical stimulation at $-60 \mathrm{mV}$ under the whole-cell patch-clamp configuration. Right: boxplots show the mean, median, and the 75th to 25th percentiles of mechano-dependent currents densities obtained by whole-cell patch-clamp recordings of scrambled or Piezo1 siRNAs transfected HMVEC. $n$ is denoted above the $x$-axis. Unpaired $t$-test. Asterisks indicate values significantly different from control $(* * * 00.001)$ and $n . s$. indicates not significantly different from the control

distribution profile similar to that of HMVEC (i.e., saturated fatty acids and $\omega-6$ PUFAS become more abundant; Fig. 6b). Under these conditions, we found a decrease in Piezol inactivation (Fig. 6c, d) and steady-state currents reminiscent of the ones measured in HMVEC (Fig. 6c, black arrow, and 6d). As AA enhances Piezol inactivation in N2A cells (Fig. 2a, b), we propose that the effect on inactivation in linoleic acid-treated cells might be due to an overall increase in linoleic acid itself and/or downstream $\omega-6$ PUFAs rather than AA (Fig. 6b). Taken together, our data support the idea that distinct Piezol gating properties, like the ones reported for N2A cells and HMVEC, are determined, in part, by the cellular fatty acid permutation.

EPA abrogates the phenotype of mutations causing xerocytosis. Mutations in human Piezol are associated with dehydrated hereditary stomatocytosis, a hemolytic anemia characterized by an increased cation permeability and a decreased osmotic fragility of erythrocytes ${ }^{16,17,44}$. For example, the alleles $\mathrm{R} 1943 \mathrm{Q}^{18}$, M2225R ${ }^{16-18}, \mathrm{R}_{2302 \mathrm{H}^{18}}, \mathrm{R} 2456 \mathrm{H}^{16,17,45}$, and R2488Q ${ }^{18,19}$ exhibit decreased inactivation when compared to wild type. In the mouse Piezol structure ${ }^{46}$, the equivalent positions to these residues are mapped in the blade (R1943), extracellular cap (M2225 and R2302), pore (R2456), and C-terminal (R2488) domains, suggesting that several regions might contribute to the inactivation process (Fig. 7a). Molecules that enhance channel inactivation or decrease activation could be useful to diminish cation permeability and erythrocyte fragility in this pathophysiological context. Given that EPA significantly decreased Piezol's time constant of inactivation in N2A cells and HMVEC (Figs. 2b and $5 \mathrm{e}$ ), we sought to determine whether EPA supplementation can recover Piezol wild-type inactivation in the frame of these slow inactivating mutants. As reported by other groups ${ }^{16-19,45}$, we found that these mutants display decreased inactivation while keeping similar displacement thresholds than the wild type (Fig. 7b-d); with the exception of mutants R2302H and R2488Q that required less displacement to activate (Fig. 7d). Remarkably, EPA treatment speeds up the time constant of inactivation of Piezo1 mutants to levels that resemble that of the wild-type channel (Fig. 7b, right panel, and Fig. 7c); as expected, EPA does not alter the activation threshold of the mutants (Fig. $7 \mathrm{~d}$ ). We transfected Piezol and mutants M2225R and R2456H (that 
a $\begin{array}{lcc}\text { Control } & \text { Margaric acid } & \text { Arachidonic acid } \\ \text { HMVEC } & \text { (MA) 17:0 } & \text { (AA) 20:4 }\end{array}$

Eicosapentaenoic acid (EPA) 20:5

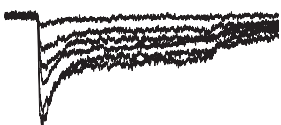

$50 \mathrm{pA}$

$$
50 \mathrm{~ms}
$$

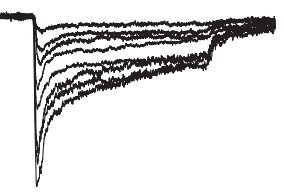

b

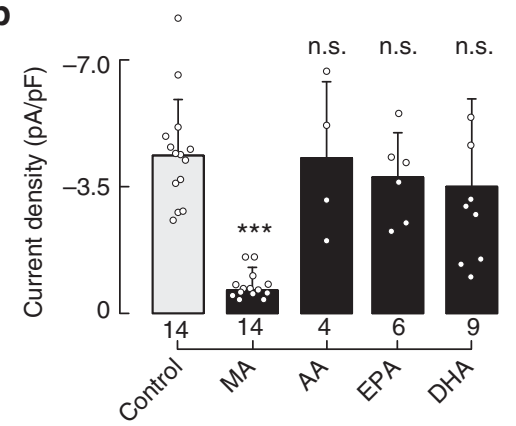

e

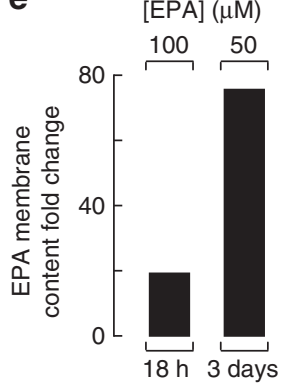

f

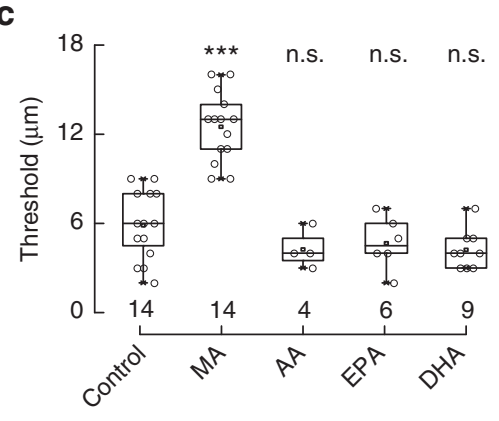

9
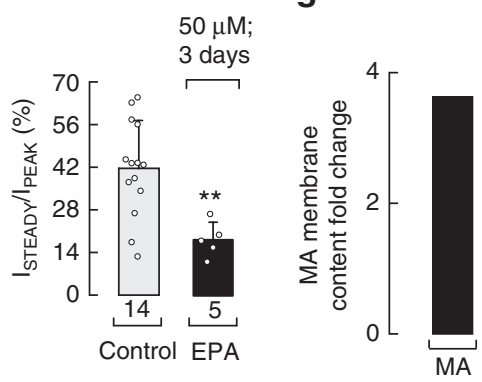

d

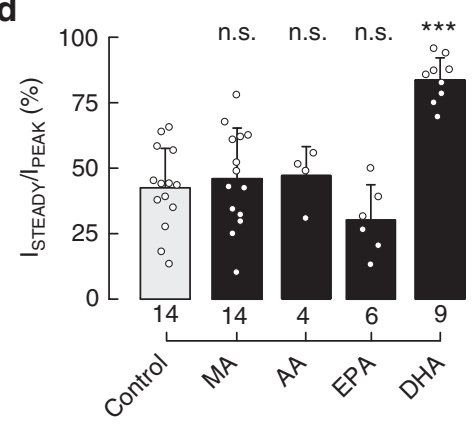

ocosahexaenoic acid (DHA) 22:6
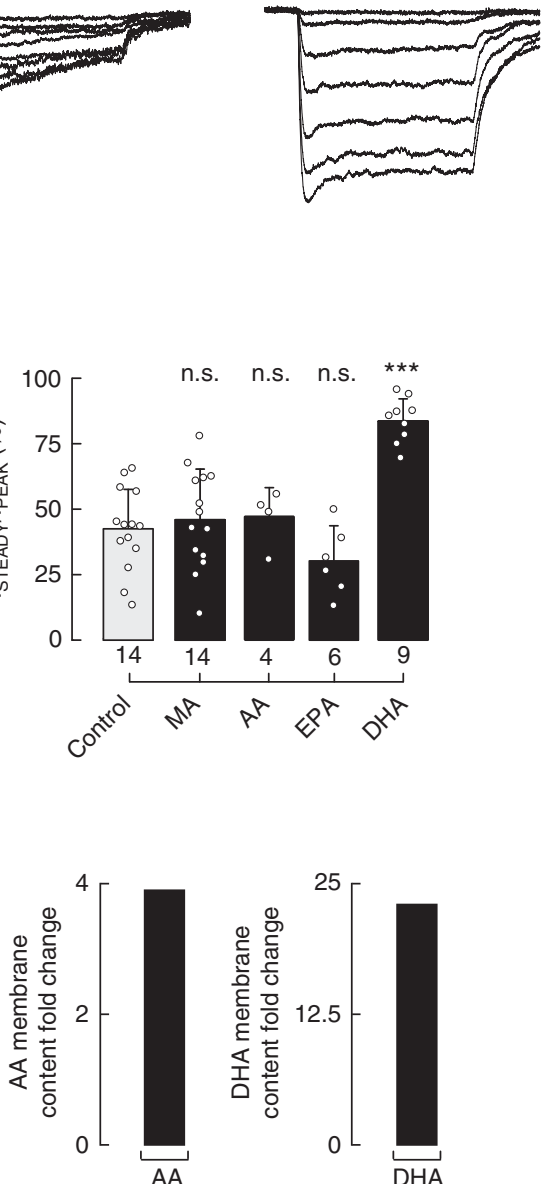

Fig. 5 Dietary fatty acids alter Piezo1 channel gating in HMVEC. a Representative whole-cell patch-clamp recordings (at -60 mV) of control, MA, AA, EPA, and DHA $(100 \mu \mathrm{M})$-treated HMVEC elicited by mechanical stimulation. b Piezo1 current densities elicited by maximum displacement of control, MA, AA, EPA, and DHA $(100 \mu \mathrm{M})$-treated HMVEC. Bars are mean \pm SD. Kruskal-Wallis and Dunn's multiple comparisons test. c Boxplots show the mean, median, and the 75 th to 25 th percentiles of the displacement threshold required to elicit Piezo1 currents of control and MA, AA, EPA, and DHA (100 $\mu M$ )-treated HMVEC. One-way ANOVA and Bonferroni test. $\mathbf{d}$ Ratio of the currents at the end of the displacement pulse to the peak current (ISTEADY/IPEAK $)$ from macroscopic traces of control, MA, AA, EPA, and DHA $(100 \mu \mathrm{M})$-treated HMVEC. Bars are mean \pm SD. One-way ANOVA and Bonferroni test. e EPA membrane content fold change of EPA-treated HMVEC supplemented with $100 \mu \mathrm{M}$ for $18 \mathrm{~h}$ and $50 \mu \mathrm{M}$ each day for 3 days, as determined by LC-MS. f Representative whole-cell patch-clamp recordings (at $-60 \mathrm{mV}$ ) of EPA ( $50 \mu \mathrm{M}$ each day for 3 days)-treated HMVEC elicited by mechanical stimulation; and ratio of the currents at the end of the displacement pulse to the peak current $\left(I_{\text {STEADY }} / I_{\text {PEAK }}\right)$ from macroscopic traces of control and EPA ( $50 \mu \mathrm{M}$ each day for three days)-treated HMVEC. Bars are mean \pm SD. Unpaired t-test. $\mathbf{g} M A, A A$, and DHA membrane content fold change in MA, AA, and DHA $(100 \mu \mathrm{M})$-treated HMVEC as determined by LC-MS. Asterisks indicate values significantly different from control $(* * p<0.001$ and $* * p<0.01)$ and $n .5$. indicates not significantly different from the control. $n$ is denoted above the $x$-axes

display the slowest inactivation time constants) in HEK-293 cells, to test whether EPA supplementation had the same effect in another cell type. As determined in N2A cells, EPA treatment decreases the time constant of inactivation in the wild-type and mutant channels expressed in HEK-293 cells (Supplementary Figure $8 \mathrm{~b}, \mathrm{c}$ ); EPA does not alter the activation threshold or current densities of the mutants (Supplementary Figure 8d-e). Altogether, these results suggest that enriching the plasma membrane with EPA overrides the effect of these gain-of-function mutations.

Fatty acids have synergistic effects on Piezol function. Our previous results demonstrate that MA modulates Piezol channel activation, whereas PUFAs alter channel inactivation. We next asked whether a combination of saturated and unsaturated fatty acids would have a synergistic effect on Piezol activity. To test this idea in endogenous Piezo1, we supplemented N2A cells with EPA and MA and found a combined effect on Piezol gating as the currents inactivated faster, were smaller, and needed more displacement for activation than the control cells (Fig. 8a-d). As dehydrated hereditary stomatocytosis mutants increased $\mathrm{Ca}^{2+}$ influx in erythrocytes, we tested the ability of the fatty acid combination to modulate the response of the gain-of-function R2456H mutant. Notably, EPA and MA supplementation reduced the inactivation time constant and current densities while increasing the displacement threshold of the $\mathrm{R} 2456 \mathrm{H}$ mutant (Fig. 8e-h). Blood plasma is a high-protein buffer as it contains $\sim 70 \mathrm{~g} \mathrm{~L}^{-1}$ of proteins; some of which bind to fatty acids. We supplemented N2A cells culture media with $70 \mathrm{~g} \mathrm{~L}^{-1}$ of BSA (the most abundant protein in human blood plasma) and found that in these conditions EPA supplementation was also efficient in 
a
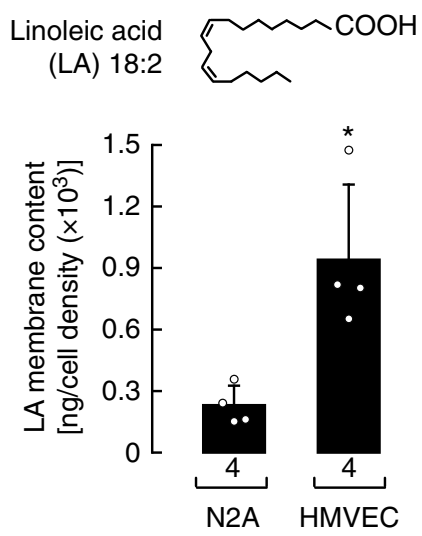

C

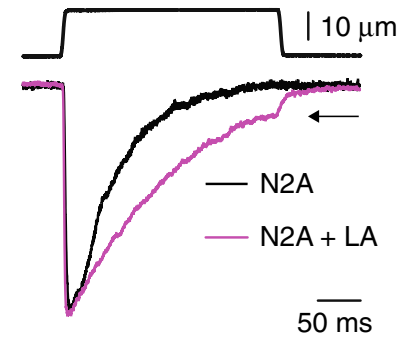

b

$\begin{array}{ll}\square \text { Monounsaturated } & \square \text { Saturated } \\ \square \omega-6 \text { PUFAs } & \square \omega-3 \text { PUFAs }\end{array}$
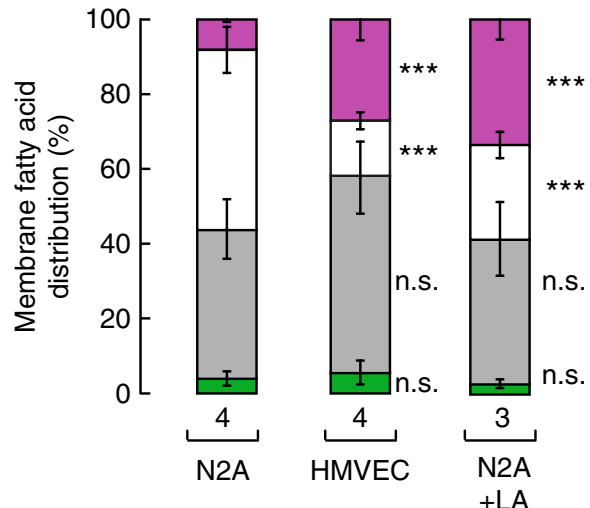

d

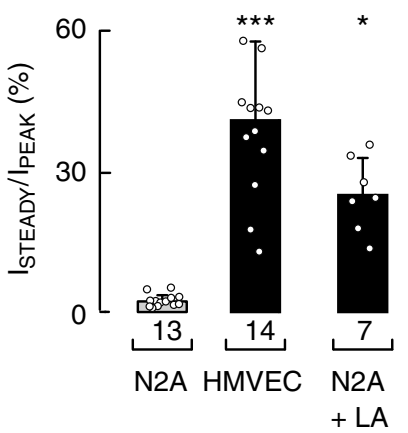

Fig. 6 Changing the cellular fatty acid distribution profile modifies Piezo1 inactivation. a Linoleic acid (LA) membrane content in N2A cells and HMVEC, as determined by LC-MS. Bars are mean \pm SD. Mann-Whitney test. b Stacked bar chart illustrating the membrane fatty acid distribution in N2A cells, HMVEC, and LA $(100 \mu \mathrm{M})$-treated N2A cells, as determined by LC-MS. Bars are mean \pm SD. One-way ANOVA and Bonferroni test. c Representative whole-cell patch-clamp recordings (at $-60 \mathrm{mV}$ ) of N2A cells and linoleic acid (LA, $100 \mu \mathrm{M}$ )-treated N2A cells elicited by mechanical stimulation. Traces were normalized for comparison. d Ratio of the currents at the end of the displacement pulse to the peak current $\left(I_{\text {STEADY }} / I_{\text {PEAK }}\right)$ from macroscopic traces of N2A cells, LA $(100 \mu \mathrm{M})$-treated cells, and HMVEC. Bars are mean \pm SD. Kruskal-Wallis and Dunn's multiple comparisons test. Asterisks indicate values significantly different from control $\left({ }^{* * *} p<0.001\right.$ and $\left.* p<0.05\right)$ and n.s. indicates not significantly different from the control. $n$ is denoted above the $x$-axis

enhancing Piezo1 inactivation (Supplementary Fig. 8f, g). As EPA and MA are biosynthesized and commonly found in fish and dairy products ${ }^{47,48}$, respectively, individual or combined fatty acids could be used as a dietary strategy to diminish the increased cation permeability observed in hemolytic anemia.

\section{Discussion}

The current model for mechanoelectrical transduction consisting of a force-bearing center seems to be universal for eukaryote cells that sense force ${ }^{49}$. In such model ion channel activity would be suppressed by a stiff platform rich in stomatin-like proteins ${ }^{50}$, the plasma membrane $\mathrm{s}^{30,31,37}$, and cytoplasmic and extracellular tethers ${ }^{13,51,52}$. Upon mechanical stimulation, the interaction between the components of this center would lead to ion channel opening, allowing a cell to convert mechanical stimuli into electrical and biochemical signals. The current knowledge of the Piezo channels suggests that they might work in a similar forcebearing center ${ }^{13}$. Importantly, the force-from-lipids principle ${ }^{53}$ establishes that the mechanical properties of the membrane control the conformational rearrangements underlying mechanosensitive ion channels gating. As fatty acids are the lipids' building blocks ${ }^{29}$ and are known to alter the mechanical properties of the membrane $e^{54,55}$, our goal was to determine the extent by which the length and unsaturation number of fatty acidcontaining lipids modify Piezol function. In this study, we present several lines of evidence that support the hypothesis that distinct fatty acid-containing phospholipids regulate Piezo1 function. First, incorporation of MA in N2A cells and HMVEC inhibits Piezol by increasing the mechanical threshold required to activate the channel. Second, decreased Piezol activation correlates with the increase in membrane rigidity determined for membranes enriched in MA. Third, long PUFAs supplementation modulates Piezol time course of inactivation. Fourth, perfusion of free fatty acids does not modify Piezol mechanical response. Fifth, Piezo1 modulation by fatty acids is not affected by disrupting the actin filaments. Sixth, Piezol exhibit different gating properties depending on the intrinsic cell fatty acid profile. Our results support the notion that fatty acid-containing phospholipids fine-tune Piezo1 mechanical response.

How do MA-containing membranes inhibit Piezol function? MA consumption has been recently associated with potential health benefits, as diets with increased daily MA intake were correlated with attenuating metabolic syndromes related to inflammation like hyperferritinemia and diabetes in dolphins and dogs, respectively ${ }^{48,56}$. Eukaryotic cell membranes are enriched in fatty acids with chain lengths from 18 to 20 carbons carrying more than one double bond ${ }^{29}$; these molecules are tightly controlled by the cell to ensure proper function of membrane proteins. We determined that augmentation of the saturated fatty acid MA in synthetic and native membranes increases structural order and rigidity. Consequently, Piezol activation requires twice the displacement to evoke a detectible response in MA-enriched membranes. Other groups have calculated that the change in 
a

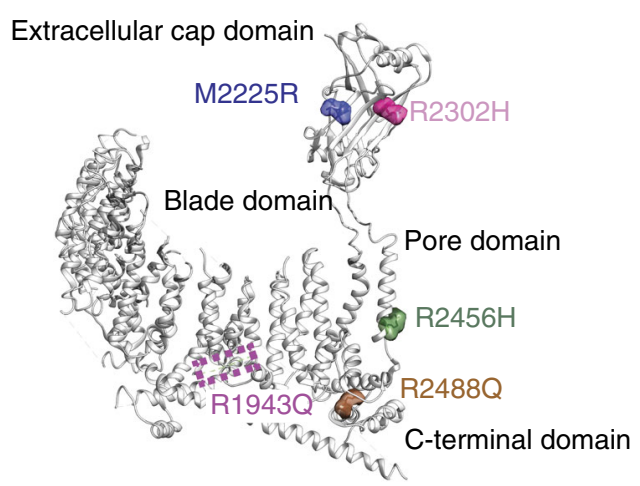

b

Control

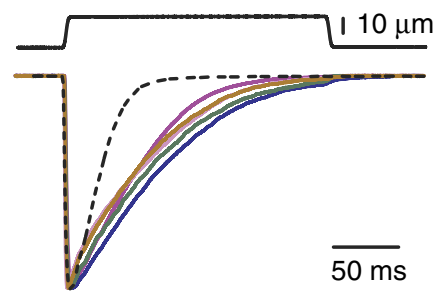

+ EPA $[200 \mu \mathrm{M}]$

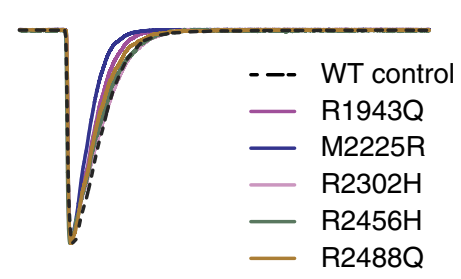

C

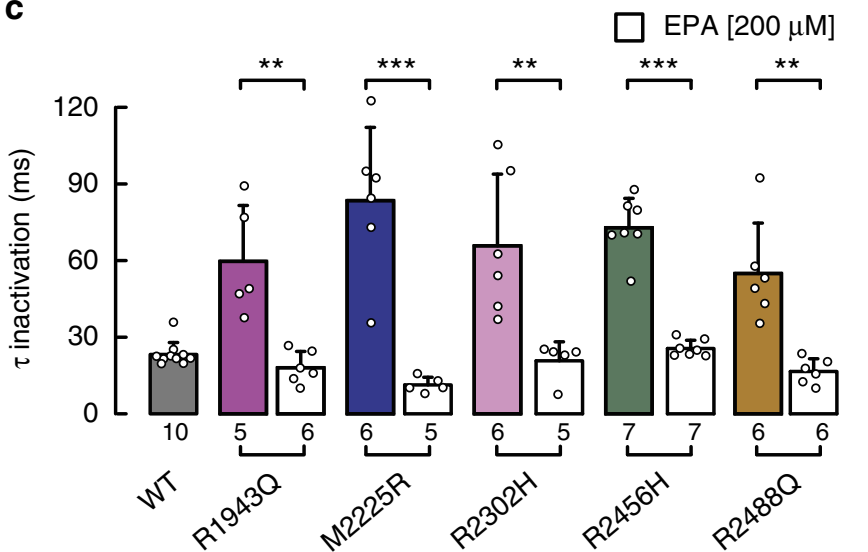

d

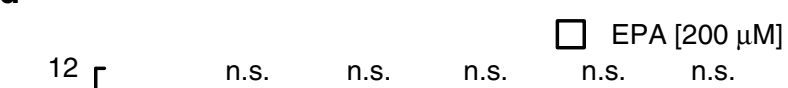

Fig. 7 EPA supplementation abrogates the phenotype of Piezo1 xerocytosis mutations. a Ribbon representation of Mus musculus (mm) Piezo1 monomer (PDB ID: 5Z10) highlighting equivalent residues that when mutated cause dehydrated hereditary stomatocytosis in humans. b Representative normalized macroscopic currents (at $-60 \mathrm{mV}$ ) evoked by maximum displacement of N2A cells transfected with human Piezo1 dehydrated hereditary stomatocytosis mutants R1943Q, M2225R, R2302H, R2456, and R2488Q with and without EPA supplementation (left and right, respectively). Human Piezo1 wild type (WT) without supplementation is shown for comparison. c Piezo1 time constants of inactivation elicited by maximum displacement of dehydrated hereditary stomatocytosis mutants R1943Q, M2225R, R2302H, R2456H, and R2488Q with and without EPA supplementation. Human Piezo1 WT without supplementation is shown for comparison. Bars are mean \pm SD. Unpaired $t$-test with Welch correction, except for R2302H in which Mann-Whitney test was used. d Boxplots show the mean, median, and the 75th to 25th percentiles of the displacement threshold required to elicit currents of Piezo1 dehydrated hereditary stomatocytosis mutants R1943Q, M2225R, R2302H, R2456H, and R2488Q with and without EPA supplementation. Human Piezo1 WT without supplementation is shown for comparison. Unpaired $t$-test, except for R2488Q in which Mann-Whitney test was used. Asterisks indicate values significantly different from control $\left({ }^{* * *} p<0.001\right.$ and $\left.{ }^{* *} p<0.01\right)$ and n.s. indicates not significantly different from the control. $n$ is denoted above the $x$-axes

Piezol in-plane area upon opening is $\sim 15 \mathrm{~nm}^{2}$ and that the tension required to activate it is $\sim 5 \mathrm{mN} \mathrm{m}^{-1} 23$. We hypothesize that the change in Piezo1 in-plane area becomes restricted during mechanical gating in the context of MA-enriched membranes, likely shifting the tension needed to activate the channel to magnitudes closer to the ones reported for the mechanosensitive channel of large conductance (MscL, $\left.\sim 12 \mathrm{mN} \mathrm{m}^{-1}\right)^{57}$. Piezol structures revealed a colossal trimer, with 38 transmembrane segments per monomer, shaped like a propeller with three blades organized around the permeation pathway $22,46,58-60$. Mutations in the long intracellular helix termed the beam reduced $47 \%$ of Piezol mechanical currents ${ }^{46}$; the beam with two structure-like hinges (latch and clasp) are thought to transmit membrane deformation from the transmembrane blades to the permeation pathway ${ }^{60}$. Rigid membranes containing MA might impair proper force transmission from the blades to the pore impairing Piezol mechanoresponse.

From other ion channel families, it is known that inactivation is due to the ion channel's intrinsic properties (e.g. $\mathrm{K}^{+}$channels C-type inactivation ${ }^{61}$ ) and/or channel-accessory subunit interactions (e.g. the $\mathrm{K}^{+}$channel Kv7.1 with $\operatorname{minK}^{62}$ ). The Piezo family is no exception, as it has been shown that Piezol inactivation is determined by the C-terminal extracellular domain
(CED) and the inner pore helix ${ }^{42,63}$. In the case of Piezo2, inactivation is modulated by a region located near the intracellular latch ${ }^{64}$. Importantly, $\mathrm{Wu}$ and collaborators demonstrated that swapping the CED between Piezo1 and Piezo2 exchanges their time courses of inactivation ${ }^{42}$. Here, we demonstrated that inactivation is also regulated by the presence of long PUFAs contained in membrane phospholipids. It is possible that PUFAs affect inactivation by modulating the allosteric coupling between the CED and the inner pore helix. Alternatively, PUFAs can alter the interaction between Piezol and other proteins. Indeed, it has been reported that Piezo1 inactivation is also modulated by TMEM150C, a transmembrane protein that belongs to a family that regulates phosphatidylinositol 4-phosphate synthesis ${ }^{65}$. In this scenario, it would be possible that plasma membranes enriched with long PUFAs alter Piezo1 modulation by TMEM150C or other proteins.

Our data shows that AA and EPA enhance Piezol inactivation, whereas DHA reduces it; this was unexpected because they all decrease plasma membrane rigidity and bending stiffness. We propose that the additional unsaturation of DHA, that is closest to the carboxylic acid group, has a larger structural effect at the membrane/channel interface, likely reducing the cohesion between neighboring lipids (reflected in the lack of cooperativity 
a

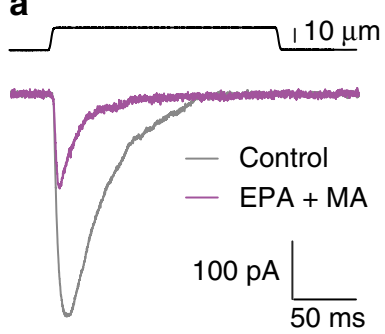

e

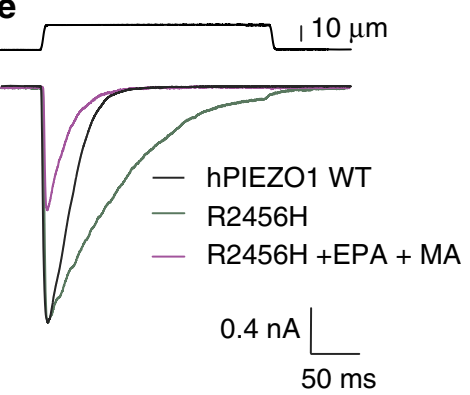

b

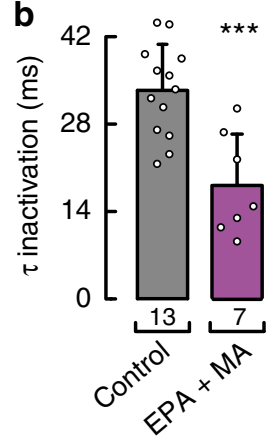

f

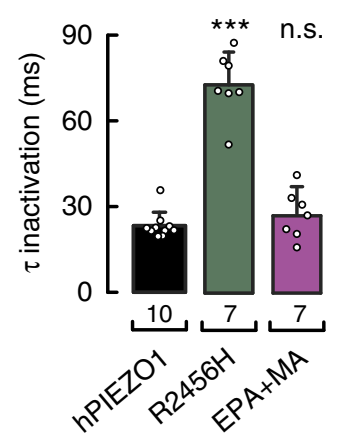

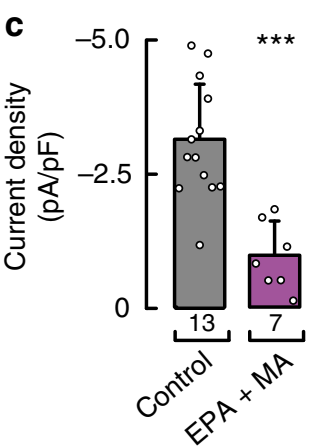

9

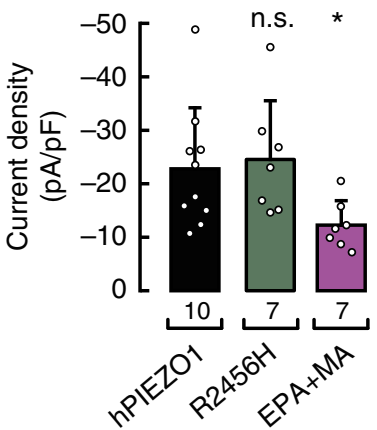

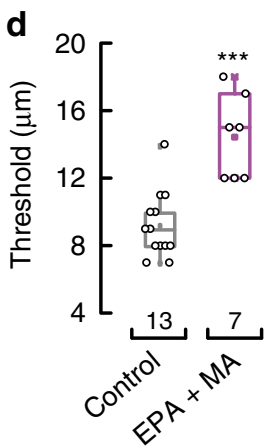

h

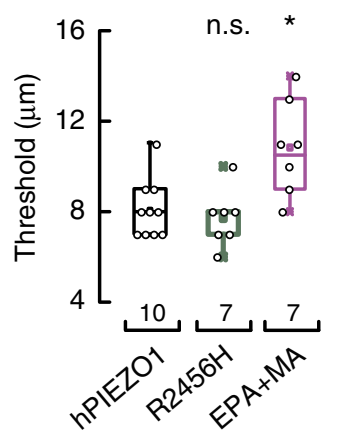

Fig. 8 EPA and MA have distinct and synergistic effects on Piezo1 gating. a Representative macroscopic current (at $-60 \mathrm{mV}$ ) evoked by maximum displacement of N2A cells treated with and without a mixture of EPA and MA (200 and $100 \mu \mathrm{M}$, respectively). b Piezo1 time constants of inactivation elicited by maximum displacement of N2A cells treated with and without a mixture of EPA and MA. Bars are mean \pm SD. One-way ANOVA and Bonferroni test. c Current densities elicited by maximum displacement of N2A cells treated with and without a mixture of EPA and MA. Bars are mean \pm SD. Unpaired t-test. d Boxplots show the mean, median, and the 75th to 25th percentiles of the displacement threshold required to elicit Piezo1 currents of N2A cells treated with and without a mixture of EPA and MA. Unpaired $t$-test. e Representative normalized macroscopic currents (at $-60 \mathrm{mV}$ ) evoked by maximum displacement of N2A cells transfected with human Piezo1 wild type (WT) and R2456H, with and without a mixture of MA and EPA supplementation (200 and $100 \mu \mathrm{M}$, respectively). f Piezo1 time constants of inactivation elicited by maximum displacement of N2A cells transfected with human Piezo1 WT and $\mathrm{R} 2456 \mathrm{H}$, with and without a mixture of MA and EPA supplementation. Bars are mean \pm SD. Kruskal-Wallis and Dunn's multiple comparisons test.

g Current densities elicited by maximum displacement of N2A cells transfected with human Piezo1 WT and R2456H, with and without a mixture of MA and EPA supplementation. Bars are mean \pm SD. Kruskal-Wallis and Dunn's multiple comparisons test. $\mathbf{h}$ Boxplots show the mean, median, and the 75th to 25th percentiles of the displacement threshold required to elicit Piezo1 currents of N2A cells transfected with human Piezo1 WT and R2456H, with and without a mixture of MA and EPA supplementation. Kruskal-Wallis and Dunn's multiple comparisons test. Asterisks indicate values significantly different from control $(* * * p<0.001$ and $* p<0.05)$ and n.s. indicates not significantly different from the control. $n$ is denoted above the $x$-axes

of the DHA's thermogram) and the transmembrane domain to stabilize the open state. Alternatively, the antagonizing effect of PUFAs could be attributed to the ability of DHA to further alter membrane organization. Indeed, it has been shown that DHA, but not EPA, has the ability to stabilize raft microdomains ${ }^{35,36,66}$. Nevertheless, the discrepancy in inactivation observed between AA and EPA with DHA remains unexplained; future in-depth studies are required to understand this effect.

Why Piezol activity changes between cell types? Piezo1 displays distinct inactivation profiles in N2A cells and HMVEC; our results supplementing N2A cells with the $\omega-6$ fatty acids precursor linoleic acid supports the idea that inactivation is modulated by the cellular fatty acid distribution. A recent work has shown that Piezol displays a non-typical slowly inactivating currents in mouse embryonic stem cells that becomes faster as the cell differentiates into motor neurons ${ }^{67}$. Importantly, there is evidence that de novo lipogenesis and changes in fatty acid synthesis, lipid metabolism, and membrane fluidity play an important role during differentiation ${ }^{66,68-70}$. For instance, differentiation of human mesenchymal stem cells into osteoblasts or adipocytes results in remodeling of the plasma membrane, yielding cell-specific membrane compositions with distinct biophysical properties ${ }^{66}$. Therefore, it remains to be determined whether the plasma membrane composition (e.g., fatty acids distribution) contributes to the different inactivation kinetics displayed by Piezo1 during cell differentiation. Taken together, the inherent cell lipid profile and dynamic changes in the fatty acid metabolism could add a level of regulation to fine-tune Piezol response to mechanical stimuli.

Several studies have shown that gain-of-function mutations in Piezol are linked to dehydrated hereditary stomatocytosis ${ }^{16-19}$, in which erythrocytes are abnormally shaped due to cation imbalance $^{19}$. Noteworthy, mutations R1942Q, M2224R, R2301H, $\mathrm{R} 2456 \mathrm{H}$, and $\mathrm{R} 2487 \mathrm{Q}$ are scattered throughout the channel (Fig. 7a); suggesting that inactivation is modulated by various regions of the protein. Our findings demonstrate that EPA supplementation abrogates the slowly inactivating phenotype of these Piezo1 mutations. EPA-enriched membranes likely exert a ubiquitous effect on the protein rather than modulating inactivation through specific binding sites. Indeed, our findings are further supported by the fact that perfusion of free EPA does not change Piezol activity. When looking for alternative supplementations protocols to counterbalance these mutations deleterious effects, we found that combining MA and EPA decreases channel currents and the time constant of inactivation of both wild type and the mutation R2456H (Fig. 8). This result supports the notion 
that fatty acids have distinct and synergistic effects on Piezol gating. Interestingly, $\omega-3$ fatty acids were used in medical trials as an effective therapy for ameliorating the symptoms associated with sickle cell anemia ${ }^{71-73}$. Hence, we envision that combining dietary fatty acids to modulate different Piezol gating modalities could be an effective therapeutic strategy to diminish the effect of hereditary mutations that alter erythrocytes' cation balance.

\section{Methods}

Cell culture and electrophysiology. Mouse neuro-2a (N2A; catalog number CCL131) and human embryonic kidney (HEK-293; catalog number CRL-1573) cell lines were obtained from the American Type Culture Collection (ATCC ${ }^{\oplus}$ ) and HMVEC (catalog number ACBRI 539) from neonatal human dermal donor isolates at passage 4 from Cell Systems Corporation. N2A, HEK-293 and HMVEC were cultured according to the manufacturer's protocol and used up to the 25th, 30 th, and 7th passage, respectively. Prior to electrophysiological measurements, N2As, HEK-293, and HMVEC were supplemented overnight $(\approx 18 \mathrm{~h})$ with $100 \mu \mathrm{M}$ (unless otherwise noted) pentadecanoic acid (PDA), heptadecanoic acid (MA) linoleic acid (LA), arachidonic acid (AA), EPA, or docosahexaenoic acid (DHA) ${ }^{31}$. For fatty acid accumulation assays, N2As were supplemented with $10 \mu \mathrm{M}$ MA or EPA every $24 \mathrm{~h}$ for 5 days; and HMVECs supplemented with $50 \mu \mathrm{M}$ EPA every 24 $\mathrm{h}$ for 3 days and used the following day. Fatty acid vials were obtained from $\mathrm{Nu}-$ Chek Prep, Inc and were freshly opened prior supplementing the cells media.

For whole-cell recordings, the bath solution contained $140 \mathrm{mM} \mathrm{NaCl}, 6 \mathrm{mM}$ $\mathrm{KCl}, 2 \mathrm{mM} \mathrm{CaCl}_{2}, 1 \mathrm{mM} \mathrm{MgCl}_{2}, 10 \mathrm{mM}$ glucose, and $10 \mathrm{mM}$ HEPES (pH 7.4). The pipette solution contained $140 \mathrm{mM} \mathrm{CsCl,} 5 \mathrm{mM}$ EGTA, $1 \mathrm{mM} \mathrm{CaCl}, 1 \mathrm{mM} \mathrm{MgCl}_{2}$, and $10 \mathrm{mM}$ HEPES (pH 7.2). Fatty acids and channel agonists and antagonists perfused during the experiments were dissolved in the bath solution: Yodal (Tocris Bioscience), GSK-417651A (FOCUS Biomolecules), HC-030031, HC-067047, and $\mathrm{GdCl}_{3}$ (Sigma-Aldrich), and L-GsMTx-4 (Abcam). Pipettes were made out of borosilicate glass (Sutter Instruments) and were fire-polished before use until a resistance between 3 and $5 \mathrm{M} \Omega$ was reached. Macroscopic currents were recorded in the whole-cell patch clamp configuration at a constant voltage $(-60 \mathrm{mV}$, unless otherwise noted), sampled at $100 \mathrm{kHz}$ and low-pass filtered at $10 \mathrm{kHz}$ using a MultiClamp 700B amplifier and Clampex (Molecular Devices, LLC). Leak currents before mechanical stimulations were subtracted off-line from the current traces and data were digitally filtered at $2 \mathrm{kHz}$ with ClampFit (Molecular Devices, LLC). Recordings with leak currents $>200 \mathrm{pA}$, with access resistance $>10 \mathrm{M} \Omega$, and cells which giga-seals did not withstand at least 6 consecutive steps of mechanical stimulation were excluded from analyses. The time constant of inactivation $\tau$ was obtained by fitting a single exponential function, Eq. (1) between the peak value of the current and the end of the stimulus:

$$
f_{(t)}=\sum_{i=1}^{n} A_{i} * e^{-t / \tau_{i}}+C
$$

where $A=$ amplitude; $\tau=$ time constant; and the constant $y$-offset $C$ for each component $i$.

Mechanical stimulation. N2As, HEK-293, and HMVEC were mechanically stimulated with a heat-polished blunt glass pipette $(3-4 \mu \mathrm{m})$ driven by a piezo servo controller (E625, Physik Instrumente). The blunt pipette was mounted on a micromanipulator at an $\sim 45^{\circ}$ angle and positioned $3-4 \mu \mathrm{m}$ above from the cells without indenting them. Displacement measurements were obtained with a squarepulse protocol consisting of $1-\mu \mathrm{m}$ incremental indentation steps, each lasting 200 $\mathrm{ms}$ with a 2 -ms ramp in 10 -s intervals. The threshold of mechano-activated currents for each experiment was defined as the indentation step that evoked the first current deflection from the baseline. For $I-V$ relationships, the holding potential was changed for $5 \mathrm{~s}$ before mechanical stimulation. Only cells that did not detach throughout stimulation protocols were included in the analysis. The piezo servo controller was automated using a MultiClamp 700B amplifier through Clampex (Molecular Devices, LLC).

Liquid chromatography-mass spectrometry. Control and fatty acid-treated N2A cells and HMVECs were cultured according to manufacturer's protocols up to 2-4 million cells. Cells were rinsed with PBS three times and frozen in liquid $\mathrm{N}_{2}$. Total and free fatty acids were extracted and quantified at the Lipidomics Core Facility at Wayne State University. Membrane (i.e., esterified) fatty acids were determined by subtracting free from total fatty acids and normalized by the number of cells in the culture. The Source Data underlying Figs. 1d, 2e, 4a, 5e, g, 6b, and Supplementary Figs. 1g, 2c, 7a are provided as a Source Data file: https://doi.org/10.6084/m9. figshare.7710140.

Differential scanning calorimetry. DSC experiments were performed as previously described ${ }^{31}$. DPPC $(4 \mathrm{mM})$ was dissolved in ethanol and mixed with specified fatty acids. Lipid mixtures containing $5 \mathrm{~mol} \%$ of individual PUFAs were evaporated. Lipid mixtures were hydrated with $10 \mathrm{mM}$ HEPES $(\mathrm{pH} 7)$ at $\approx 50^{\circ} \mathrm{C}$. To yield multilamellar vesicles, the suspension was vortexed and incubated for $1 \mathrm{~h}$ at $\approx 50{ }^{\circ} \mathrm{C}$. Samples were degassed at $635 \mathrm{mmHg}$ for $10 \mathrm{~min}$ at $3^{\circ} \mathrm{C}$ and equilibrated for $5 \mathrm{~min}$ at $4{ }^{\circ} \mathrm{C}$ prior to DSC experiments. Measurements were performed on a NanoDSC microcalorimeter (TA Instruments). Thermograms were recorded at a constant scan rate of $1^{\circ} \mathrm{C} \mathrm{min}-1$ (between 10 and $60{ }^{\circ} \mathrm{C}$ ), and a pressure of $3 \mathrm{~atm}$, and were analyzed with Nano Analyze.

Atomic force microscopy. N2A cells were supplemented with $100 \mu \mathrm{M}$ fatty acids overnight $(\approx 18 \mathrm{~h})$ prior to measurements. To disrupt the cytoskeleton, control and fatty acid supplemented cells were incubated with $1 \mu \mathrm{M}$ latrunculin A (Cayman Chemicals) for $1 \mathrm{~h}$, as established elsewhere ${ }^{40}$. AFM experiments were carried out as previously described ${ }^{31,74}$ with a Bruker BioScope Resolve ${ }^{\text {tx }}$ atomic force microscope using the contact mode. In brief, AFM cantilevers MLCT-Bio-DC A probe (Bruker) were coated for $1 \mathrm{~h}$ with $1 \mathrm{mg} \mathrm{ml}^{-1}$ peanut lectin in PBS ( $\mathrm{pH} 7.4$ ) and calibrated per the thermal noise method ${ }^{75}$ with spring constants of $55-65 \mathrm{pN} \mathrm{nm}^{-1}$. Membrane tethers were pulled at $40 \mu \mathrm{m} \mathrm{s}^{-1}$ from N2A cells at $25^{\circ} \mathrm{C}$. To optimize the number of bonds between the peanut-lectin coated cantilever and the membrane, the contact force was $3.26 \mathrm{nN}$ while the contact time was $750 \mathrm{~ms}$. Individual ramp scans were used to generate force-distance curves displaying unbinding events. For cellcantilever contacts that yielded multiple-tether events, forces were characterized by the length of the last observed ruptured tether ${ }^{39}$; membrane tethers refer to the length of ruptured events (Supplementary Figure 3a). Data were obtained from at least three tissue culture plates ( $\geq 25$ cells per plate), acquired and analyzed with NanoScope (Bruker Corporation) and presented using estimation plots ${ }^{76}$. Data points $>200 \mathrm{pN}$ were excluded.

Calcium imaging. HMVEC were loaded with Fluo-4 AM (1 mM; Invitrogen) per the manufacturer's protocols. Images were acquired and analyzed with CellSens Dimensions (Olympus Corp.). The solution contained $140 \mathrm{mM} \mathrm{NaCl}, 2 \mathrm{mM} \mathrm{CaCl}_{2}$, $1 \mathrm{mM} \mathrm{MgCl}$, $10 \mathrm{mM}$ HEPES, $10 \mathrm{mM}$ glucose, and $20 \mathrm{mM}$ mannitol (320 mOsm) ( $\mathrm{pH}$ 7.3). Increases in fluorescence were achieved by perfusing $2 \mu \mathrm{M}$ of Yodal (Tocris Bioscience) and washed with control solution. Cells with a large baseline fluorescence signal ( $>20$ arbitrary units) were excluded from the analysis.

siRNA-mediated knockdown and q-RT-PCR. HMVEC were transfected with Lipofectamine $^{\circledast}$ RNAiMAX Transfection Reagent (Thermo Fisher Scientific) according the manufacturer's protocols ${ }^{1}$. The siRNA concentration was $20 \mathrm{nM}$ for Piezol and silencer negative control (Thermo Fisher Scientific). The transfection was done with free-antibiotic media and after $6 \mathrm{~h}$ of transfection, the medium was replaced with a fresh one containing antibiotics. For electrophysiology experiments, cells were also co-transfected with siGLO Green Transfection Indicator (Dhar$\left.\operatorname{macon}^{\mathrm{Tw}}\right)$. The targeted sequences of siRNAs directed against human Piezol RNA (ThermoFisher Scientific) was: 5'-AAGAAGAUCGUCAAGUACG-3'. The negative control (scrambled siRNA) was purchased from Ambion, Inc.

RNAs were isolated from HMVEC using RNeasy ${ }^{\circledR}$ Mini Kit (QIAGEN). cDNA was generated with iScript ${ }^{\text {tw }}$ Reverse Transcription Supermix for RT-qPCR (BioRad). qPCR triplicate reactions were run in a CFX Connect ${ }^{\text {tu }}$ Real-Time PCR detection system with SsoAdvanced ${ }^{\text {mt }}$ Universal SYBR ${ }^{\circledast}$ Green supermix (Bio-Rad) according to the manufacturer's instructions. Data acquisition and analysis was done using Bio-Rad CFX Maestro 1.0 (Bio-Rad Laboratories). Normalization was done using $\Delta \Delta \mathrm{Cq}$ with GAPDH as the reference gene. Standard curves were generated from specific templates for each PrimePCR ${ }^{\mathrm{rm}}$ assay mentioned below. Primers were purchased from Bio-Rad for Human GAPDH (Unique Assay ID: qHsaCED0038674) and hPiezol (Unique Assay ID: qHsaCID0012344).

N2A and HEK-293 cells transfection. N2A and HEK-293 cells were transfected with $1 \mu \mathrm{g}$ of human PIEZO1 wild type and mutants (R1943Q, M2225R, R2302H $\mathrm{R} 2456 \mathrm{H}$, and R2488Q), cloned in pIRES2-EGFP ${ }^{45}$, using Lipofectamine 2000 (Thermo Fisher Scientific) according to the manufacturer's instructions and recorded $48 \mathrm{~h}$ later. Overexpression of Piezol wild-type and gain-of-function mutants elicited currents that were 10-times higher than the Piezol native ones (Supplementary Figure 8a). Fatty acids were supplemented 18-24 h prior recording.

Data analysis. Results were expressed as means \pm SD (unless otherwise noted). Box plots depict a range between the 25th and 75th percentiles, mean, median, and outliers with a 1.5 coefficient. Data were plotted using OriginPro (from OriginLab) and QtiPlot (from Ion Vasilief). Sigmoidal fitting was done using OriginPro with the following Boltzmann function:

$$
f_{(x)}=A_{2}+\frac{A_{1}-A_{2}}{1+e^{\left(\left(X-X_{0}\right) / d X\right)}}
$$

where $A_{2}=$ final value; $A_{1}=$ initial value; $X_{\mathrm{o}}=$ center; and $\mathrm{d} X=$ time constant

Statistical analyses were performed using GraphPad Instat 3 software. One-way ANOVA with Bonferroni test, repeated measures analysis of variance with Bonferroni test, two-tailed paired and unpaired $t$-test were used to evaluate statistical significance between samples. When the SD's of the tested groups were significantly different, and/or the data were not sampled from a normal distribution, the statistical significances were evaluated with Kruskal-Wallis with Dunn's test, Friedman's test with Dunn's test, or Mann-Whitney test. Individual tests are described on each of the Figure legends. 
Reporting summary. Further information on experimental design is available in the Nature Research Reporting Summary linked to this article.

\section{Data availability}

Data supporting the findings of this manuscript are available from the corresponding author upon reasonable request. A reporting summary for this Article is available as a Supplementary Information file. The Source Data underlying Figs. 1b-g, 2b-f, 3b-d, $4 a$, $4 c-e, 5 b-f$, and 6b, 6d, 7c, d, 8b-d, 8f-h, and Supplementary Figs. 1a, b, 1d-g, 2b-d, 3b, $3 \mathrm{~d}-\mathrm{f}, 4 \mathrm{a}, \mathrm{b}, 5 \mathrm{a}, 5 \mathrm{f}, 6 \mathrm{a}, \mathrm{b}, 7 \mathrm{a}, 8 \mathrm{a}, 8 \mathrm{c}-\mathrm{e}, 8 \mathrm{~g}$ are provided as a Source Data file.

Received: 1 October 2018 Accepted: 18 February 2019

Published online: 13 March 2019

\section{References}

1. Wang, S. et al. Endothelial cation channel PIEZO1 controls blood pressure by mediating flow-induced ATP release. J. Clin. Invest. 126, 4527-4536 (2016).

2. Rode, B. et al. Piezol channels sense whole body physical activity to rese cardiovascular homeostasis and enhance performance. Nat. Commun. 8, 350 (2017).

3. Zeng, W. Z. et al. PIEZOs mediate neuronal sensing of blood pressure and the baroreceptor reflex. Science 362, 464-467 (2018).

4. Ranade, S. S. et al. Piezo1, a mechanically activated ion channel, is required for vascular development in mice. Proc. Natl Acad. Sci. USA 111, 10347-10352 (2014).

5. Li, J. et al. Piezol integration of vascular architecture with physiological force. Nature 515, 279-282 (2014).

6. Cahalan, S. M. et al. Piezol links mechanical forces to red blood cell volume. Elife 4, e07370 (2015).

7. Servin-Vences, M. R., Moroni, M., Lewin, G. R. \& Poole, K. Direct measurement of TRPV4 and PIEZO1 activity reveals multiple mechanotransduction pathways in chondrocytes. Elife 6, e21074 (2017).

8. Lee, W. et al. Synergy between Piezo1 and Piezo2 channels confers high-strain mechanosensitivity to articular cartilage. Proc. Natl Acad. Sci. USA 111, E5114-E5122 (2014)

9. Pathak, M. M. et al. Stretch-activated ion channel Piezol directs lineage choice in human neural stem cells. Proc. Natl Acad. Sci. USA 111, 16148-16153 (2014).

10. Blumenthal, N. R., Hermanson, O., Heimrich, B. \& Shastri, V. P. Stochastic nanoroughness modulates neuron-astrocyte interactions and function via mechanosensing cation channels. Proc. Natl Acad. Sci. USA 111, 16124-16129 (2014).

11. Wu, J., Lewis, A. H. \& Grandl, J. Touch, tension, and transduction - the function and regulation of piezo ion channels. Trends Biochem. Sci. 42, 57-71 (2017).

12. $\mathrm{Ma}, \mathrm{S}$. et al. Common PIEZO1 allele in African populations causes RBC dehydration and attenuates plasmodium infection. Cell 173, 443-455 (2018).

13. Nourse, J. L. \& Pathak, M. M. How cells channel their stress: interplay between Piezol and the cytoskeleton. Semin. Cell Dev. Biol. 71, 3-12 (2017).

14. Martin-Almedina, S., Mansour, S. \& Ostergaard, P. Human phenotypes caused by PIEZO1 mutations; one gene, two overlapping phenotypes? J. Physiol. 596, 985-992 (2018).

15. Alper, S. L. Genetic diseases of PIEZO1 and PIEZO2 dysfunction. Curr. Top. Membr. 79, 97-134 (2017).

16. Zarychanski, R. et al. Mutations in the mechanotransduction protein PIEZO1 are associated with hereditary xerocytosis. Blood 120, 1908-1915 (2012).

17. Albuisson, J. et al. Dehydrated hereditary stomatocytosis linked to gain-offunction mutations in mechanically activated PIEZO1 ion channels. Nat. Commun. 4, 1884 (2013).

18. Glogowska, E. et al. Novel mechanisms of PIEZO1 dysfunction in hereditary xerocytosis. Blood 130, 1845-1856 (2017).

19. Andolfo, I. et al. Multiple clinical forms of dehydrated hereditary stomatocytosis arise from mutations in PIEZO1. Blood 121, 3925-3935 (2013). S1-12.

20. Bae, C., Sachs, F. \& Gottlieb, P. A. The mechanosensitive ion channel Piezol is inhibited by the peptide GsMTx4. Biochemistry 50, 6295-6300 (2011).

21. Lewis, A. H. \& Grandl, J. Mechanical sensitivity of Piezol ion channels can be tuned by cellular membrane tension. Elife 4, e12088 (2015).

22. Guo, Y. R. \& MacKinnon, R. Structure-based membrane dome mechanism for Piezo mechanosensitivity. Elife 6, e33660 (2017).

23. Cox, C. D. et al. Removal of the mechanoprotective influence of the cytoskeleton reveals PIEZO1 is gated by bilayer tension. Nat. Commun. 7, 10366 (2016)

24. Syeda, R. et al. Piezol channels are inherently mechanosensitive. Cell Rep. 17, 1739-1746 (2016).
25. Borbiro, I., Badheka, D. \& Rohacs, T. Activation of TRPV1 channels inhibits mechanosensitive Piezo channel activity by depleting membrane phosphoinositides. Sci. Signal. 8, ra15 (2015).

26. Tsuchiya, M. et al. Cell surface flip-flop of phosphatidylserine is critical for PIEZO1-mediated myotube formation. Nat. Commun. 9, 2049 (2018).

27. Cordero-Morales, J. F. \& Vasquez, V. How lipids contribute to ion channel function, a fat perspective on direct and indirect interactions. Curr. Opin. Struct. Biol. 51, 92-98 (2018).

28. Elinder, F. \& Liin, S. I. Actions and Mechanisms of polyunsaturated fatty acids on voltage-gated ion channels. Front. Physiol. 8, 43 (2017).

29. Mouritsen, O. G. Life - As A Matter Of Fat: The Emerging Science of Lipidomics, 276 (WILEY-VCH Verlag GmbH \& Co. KGaA, Weinheim, 2004).

30. Randall, A. S. et al. Speed and sensitivity of phototransduction in Drosophila depend on degree of saturation of membrane phospholipids. J. Neurosci. 35, 2731-2746 (2015).

31. Caires, R. et al. Omega-3 fatty acids modulate TRPV4 function through plasma membrane remodeling. Cell Rep. 21, 246-258 (2017).

32. Coste, B. et al. Piezol and Piezo2 are essential components of distinct mechanically activated cation channels. Science 330, 55-60 (2010).

33. Abdelmagid, S. A. et al. Comprehensive profiling of plasma fatty acid concentrations in young healthy Canadian adults. PLoS ONE 10, e0116195 (2015).

34. Syeda, R. et al. Chemical activation of the mechanotransduction channel Piezo1. Elife 4, e07369 (2015).

35. Mason, R. P., Jacob, R. F., Shrivastava, S., Sherratt, S. C. R. \& Chattopadhyay, A. Eicosapentaenoic acid reduces membrane fluidity, inhibits cholesterol domain formation, and normalizes bilayer width in atherosclerotic-like model membranes. Biochim. Biophys. Acta 1858, 3131-3140 (2016).

36. Sherratt, S. C. R. \& Mason, R. P. Eicosapentaenoic acid and docosahexaenoic acid have distinct membrane locations and lipid interactions as determined by X-ray diffraction. Chem. Phys. Lipids 212, 73-79 (2018).

37. Vasquez, V., Krieg, M., Lockhead, D. \& Goodman, M. B. Phospholipids that contain polyunsaturated fatty acids enhance neuronal cell mechanics and touch sensation. Cell Rep. 6, 70-80 (2014).

38. Bruno, M. J., Koeppe, R. E. 2nd \& Andersen, O. S. Docosahexaenoic acid alters bilayer elastic properties. Proc. Natl Acad. Sci. USA 104, 9638-9643 (2007).

39. Sun, M. et al. Multiple membrane tethers probed by atomic force microscopy. Biophys. J. 89, 4320-4329 (2005).

40. Krieg, M., Dunn, A. R. \& Goodman, M. B. Mechanical control of the sense of touch by beta-spectrin. Nat. Cell Biol. 16, 224-233 (2014).

41. O'Hagan, R., Chalfie, M. \& Goodman, M. B. The MEC-4 DEG/ENaC channel of Caenorhabditis elegans touch receptor neurons transduces mechanical signals. Nat. Neurosci. 8, 43-50 (2005).

42. Wu, J. et al. Inactivation of mechanically activated Piezol ion channels is determined by the C-terminal extracellular domain and the inner pore helix. Cell Rep. 21, 2357-2366 (2017)

43. Rett, B. S. \& Whelan, J. Increasing dietary linoleic acid does not increase tissue arachidonic acid content in adults consuming Western-type diets: a systematic review. Nutr. Metab. 8, 36 (2011).

44. Delaunay, J. The hereditary stomatocytoses: genetic disorders of the red cell membrane permeability to monovalent cations. Semin. Hematol. 41, 165-172 (2004).

45. Bae, C., Gnanasambandam, R., Nicolai, C., Sachs, F. \& Gottlieb, P. A. Xerocytosis is caused by mutations that alter the kinetics of the mechanosensitive channel PIEZO1. Proc. Natl Acad. Sci. USA 110 E1162-E1168 (2013).

46. Zhao, Q. et al. Structure and mechanogating mechanism of the Piezol channel. Nature 554, 487-492 (2018).

47. Sayanova, O. V. \& Napier, J. A. Eicosapentaenoic acid: biosynthetic routes and the potential for synthesis in transgenic plants. Phytochemistry 65, 147-158 (2004).

48. Jenkins, B. J. et al. Odd chain fatty acids; new insights of the relationship between the gut microbiota, dietary intake, biosynthesis and glucose intolerance. Sci. Rep. 7, 44845 (2017).

49. Anishkin, A. \& Kung, C. Stiffened lipid platforms at molecular force foci. Proc Natl Acad. Sci. USA 110, 4886-4892 (2013).

50. Poole, K., Herget, R., Lapatsina, L., Ngo, H. D. \& Lewin, G. R. Tuning Piezo ion channels to detect molecular-scale movements relevant for fine touch. Nat. Commun. 5, 3520 (2014).

51. Assad, J. A., Shepherd, G. M. \& Corey, D. P. Tip-link integrity and mechanical transduction in vertebrate hair cells. Neuron 7, 985-994 (1991).

52. Hu, J., Chiang, L. Y., Koch, M. \& Lewin, G. R. Evidence for a protein tether involved in somatic touch. EMBO J. 29, 855-867 (2010).

53. Teng, J., Loukin, S., Anishkin, A. \& Kung, C. The force-from-lipid (FFL) principle of mechanosensitivity, at large and in elements. Pflugers Arch. 467, 27-37 (2015) 
54. Rawicz, W., Olbrich, K. C., McIntosh, T., Needham, D. \& Evans, E. Effect of chain length and unsaturation on elasticity of lipid bilayers. Biophys. J. 79, 328-339 (2000).

55. Rajamoorthi, K., Petrache, H. I., McIntosh, T. J. \& Brown, M. F. Packing and viscoelasticity of polyunsaturated omega- 3 and omega- 6 lipid bilayers as seen by (2)H NMR and X-ray diffraction. J. Am. Chem. Soc. 127, 1576-1588 (2005).

56. Venn-Watson, S. K. et al. Increased dietary intake of saturated fatty acid heptadecanoic acid (C17:0) associated with decreasing ferritin and alleviated metabolic syndrome in dolphins. PLoS ONE 10, e0132117 (2015).

57. Sukharev, S. I., Sigurdson, W. J., Kung, C. \& Sachs, F. Energetic and spatial parameters for gating of the bacterial large conductance mechanosensitive channel, MscL. J. Gen. Physiol. 113, 525-540 (1999).

58. Ge, J. et al. Architecture of the mammalian mechanosensitive Piezol channel. Nature 527, 64-69 (2015).

59. Saotome, K. et al. Structure of the mechanically activated ion channel Piezol. Nature 554, 481-486 (2018).

60. Chesler, A. T. \& Szczot, M. Piezo ion channels: portraits of a pressure sensor. Elife 7, e34396 (2018).

61. Armstrong, C. M. \& Hollingworth, S. A perspective on Na and K channel inactivation. J. Gen. Physiol. 150, 7-18 (2018).

62. Tristani-Firouzi, M. \& Sanguinetti, M. C. Voltage-dependent inactivation of the human $\mathrm{K}+$ channel KvLQT1 is eliminated by association with minimal $\mathrm{K}+$ channel (minK) subunits. J. Physiol. 510 (Pt 1), 37-45 (1998).

63. Zheng, W., Gracheva, E. O. \& Bagriantsev, S. N. A hydrophobic gate in the inner pore helix is the major determinant of inactivation in mechanosensitive Piezo channels. Elife 8, e44003 (2019).

64. Szczot, M. et al. Cell-type-specific splicing of Piezo2 regulates mechanotransduction. Cell Rep. 21, 2760-2771 (2017).

65. Anderson, E. O., Schneider, E. R., Matson, J. D., Gracheva, E. O. \& Bagriantsev, S. N. TMEM150C/Tentonin3 is a regulator of mechano-gated ion channels. Cell Rep. 23, 701-708 (2018).

66. Levental, K. R. et al. Omega-3 polyunsaturated fatty acids direct differentiation of the membrane phenotype in mesenchymal stem cells to potentiate osteogenesis. Sci. Adv. 3, eaaol193 (2017).

67. Del Marmol, J. I., Touhara, K. K., Croft, G. \& MacKinnon, R. Piezo1 forms a slowly-inactivating mechanosensory channel in mouse embryonic stem cells. Elife 7, e33149 (2018).

68. Knobloch, M. et al. Metabolic control of adult neural stem cell activity by Fasn-dependent lipogenesis. Nature 493, 226-230 (2013).

69. Matsuzaki, T. et al. Defining lineage-specific membrane fluidity signatures that regulate adhesion kinetics. Stem Cell Rep. 11, 852-860 (2018).

70. Kiamehr, M. et al. Lipidomic profiling of patient-specific iPSC-derived hepatocyte-like cells. Dis. Model Mech. 10, 1141-1153 (2017).

71. Ataga, K. I. New and experimental agents for sickle cell disease. Clin. Adv. Hematol. Oncol. 16, 476-479 (2018).

72. Daak, A. A. et al. Effect of omega-3 (n-3) fatty acid supplementation in patients with sickle cell anemia: randomized, double-blind, placebo-controlled trial. Am. J. Clin. Nutr. 97, 37-44 (2013).

73. Daak, A. A. et al. Double-blind, randomized, multicenter phase 2 study of SC411 in children with sickle cell disease (SCOT trial). Blood Adv. 2, 1969-1979 (2018).

74. Krieg, M., Helenius, J., Heisenberg, C. P. \& Muller, D. J. A bond for a lifetime: employing membrane nanotubes from living cells to determine receptorligand kinetics. Angew. Chem. Int. Ed. Engl. 47, 9775-9777 (2008).

75. Hutter, J. L. \& Bechhoefer, J. Calibration of atomic-force microscope tips. Rev. Sci. Instrum. 64, 1868-1873 (1993).
76. Ho, J., Tumkaya, T., Aryal, S., Choi, H. \& Claridge-Chang, A. Moving beyond P values: everyday data analysis with estimation plots. Preprint at https:// www.biorxiv.org/content/10.1101/377978v1 (2018).

\section{Acknowledgements}

We thank Dr. J.C. Ruiz-Suárez for providing access to the DSC instrument; Dr. P.A Gottlieb for providing human Piezo1 R2456H in pIRES2-EGFP; and Dr. A. Chesler for technical advice and critically reading the manuscript; Dr. C.M. Waters and Dr. R.C. Foehring for critically reading the manuscript; and members of the Cordero and Vásquez laboratories for technical support. We used the lipidomics Core Facility at Wayne State University (NIH S10RR027926). This work was supported by the National Institutes of Health (R01GM125629 to J.F.C.-M.; and R01CA210192, R01CA206069, and R01CA204552 to S.C.C.), the United States-Israel Binational Science Foundation (2015221 to V.V.), and the American Heart Association (16SDG26700010 to V.V. and 15 SDG25700146 to J.F.C.-M.).

\section{Author contributions}

Conceptualization: V.V.; methodology: V.V., J.F.C.-M. and L.O.R.; formal analysis: V.V. and L.O.R.; investigation: L.O.R., F.J.S.-V., A.E.M. and A.D.M.-D.; resources: V.V., J.F. C.-M. and S.C.C., writing - original draft preparation: V.V.; writing - review \& editing: V.V. and J.F.C.-M.; supervision: V.V. and J.F.C.-M.; project administration: V.V.; funding acquisition: V.V. and J.F.C.-M.

\section{Additional information}

Supplementary Information accompanies this paper at https://doi.org/10.1038/s41467 019-09055-7.

Competing interests: The authors declare no competing interests.

Reprints and permission information is available online at http://npg.nature.com/ reprintsandpermissions/

Journal peer review information: Nature Communications thanks Benjamin Gaub, Tibor Rohacs and the other anonymous reviewer(s) for their contribution to the peer review of this work. Peer reviewer reports are available.

Publisher's note: Springer Nature remains neutral with regard to jurisdictional claims in published maps and institutional affiliations.

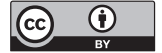

Open Access This article is licensed under a Creative Commons Attribution 4.0 International License, which permits use, sharing, adaptation, distribution and reproduction in any medium or format, as long as you give appropriate credit to the original author(s) and the source, provide a link to the Creative Commons license, and indicate if changes were made. The images or other third party material in this article are included in the article's Creative Commons license, unless indicated otherwise in a credit line to the material. If material is not included in the article's Creative Commons license and your intended use is not permitted by statutory regulation or exceeds the permitted use, you will need to obtain permission directly from the copyright holder. To view a copy of this license, visit http://creativecommons.org/ licenses/by/4.0/

(c) The Author(s) 2019 